\title{
Extended Matrix Variate Hypergeometric Functions and Matrix Variate Distributions
}

\author{
Daya K. Nagar, ${ }^{1}$ Raúl Alejandro Morán-Vásquez, ${ }^{1}$ and Arjun K. Gupta ${ }^{2}$ \\ ${ }^{1}$ Instituto de Matemáticas, Universidad de Antioquia, Calle 67, No. 53-108, Medellín, Colombia \\ ${ }^{2}$ Department of Mathematics and Statistics, Bowling Green State University, Bowling Green, OH 43403-0221, USA
}

Correspondence should be addressed to Daya K. Nagar; dayaknagar@yahoo.com

Received 7 July 2014; Accepted 22 December 2014

Academic Editor: Biren N. Mandal

Copyright (C) 2015 Daya K. Nagar et al. This is an open access article distributed under the Creative Commons Attribution License, which permits unrestricted use, distribution, and reproduction in any medium, provided the original work is properly cited.

Hypergeometric functions of matrix arguments occur frequently in multivariate statistical analysis. In this paper, we define and study extended forms of Gauss and confluent hypergeometric functions of matrix arguments and show that they occur naturally in statistical distribution theory.

\section{Introduction}

The classical beta function, denoted by $B(a, b)$, is defined (Luke [1]) by the integral

$$
B(a, b)=\int_{0}^{1} t^{a-1}(1-t)^{b-1} d t, \quad \operatorname{Re}(a)>0, \operatorname{Re}(b)>0 .
$$

Based on the beta function, the Gauss hypergeometric function, denoted by $F(a, b ; c ; z)$, and the confluent hypergeometric function, denoted by $\Phi(b ; c ; z)$, for $\operatorname{Re}(c)>\operatorname{Re}(b)>0$, are defined as (Luke [1])

$$
\begin{aligned}
F(a, b ; c ; z)= & \frac{1}{B(b, c-b)} \int_{0}^{1} \frac{t^{b-1}(1-t)^{c-b-1}}{(1-z t)^{a}} d t, \\
\Phi(b ; c ; z)= & \frac{1}{B(b, c-b)} \\
& \times \int_{0}^{1} t^{b-1}(1-t)^{c-b-1} \exp (z t) d t .
\end{aligned}
$$

Further, using the series expansions of $(1-z t)^{-a},|z t|<1$, and $\exp (z t)$ in (2) and (3), respectively, series representations of hypergeometric functions $F$ and $\Phi$, for $\operatorname{Re}(c)>\operatorname{Re}(b)>0$, are obtained as

$$
\begin{gathered}
F(a, b ; c ; z)=\sum_{n=0}^{\infty} \frac{(a)_{n} B(b+n, c-b)}{B(b, c-b)} \frac{z^{n}}{n !}, \quad|z|<1, \\
\Phi(b ; c ; z)=\sum_{n=0}^{\infty} \frac{B(b+n, c-b)}{B(b, c-b)} \frac{z^{n}}{n !},
\end{gathered}
$$

respectively.

From the confluent hypergeometric function $\Phi(b ; c ; z)$, the Whittaker function (Whittaker and Watson [2]) $M_{k, \mu}(z)$ is defined as

$$
\begin{aligned}
M_{k, \mu}(z)=z^{\mu+1 / 2} \exp & \left(-\frac{z}{2}\right) \\
& \times \Phi\left(\mu-k+\frac{1}{2} ; 2 \mu+1 ; z\right),
\end{aligned}
$$

where $\operatorname{Re}(\mu \pm k)>-1 / 2$. Most of the properties and integral representations of the Whittaker function can be proved from those of the confluent hypergeometric function.

In 1997, Chaudhry et al. [3] extended the classical beta function to the whole complex plane by introducing in the integrand of (1) the exponential factor $\exp [-\sigma / t(1-t)]$ with $\operatorname{Re}(\sigma)>0$. Thus, the extended beta function is defined as

$$
B(a, b ; \sigma)=\int_{0}^{1} t^{a-1}(1-t)^{b-1} \exp \left[-\frac{\sigma}{t(1-t)}\right] d t,
$$


where $\operatorname{Re}(\sigma)>0$. If we take $\sigma=0$ in (7), then for $\operatorname{Re}(a)>0$ and $\operatorname{Re}(b)>0$ we have $B(a, b ; 0)=B(a, b)$. Further, replacing $t$ by $1-t$ in (7), one can see that $B(a, b ; \sigma)=B(b, a ; \sigma)$. The rationale and justification for introducing this function are given in Chaudhry et al. [3] where several properties and a statistical application have also been studied. Miller [4] further studied this function and has given several additional results.

In 2004, Chaudhry et al. [5] presented definitions of the extended Gauss hypergeometric function and the extended confluent hypergeometric function, denoted by $F_{\sigma}(a, b ; c ; z)$ and $\Phi_{\sigma}(b ; c ; z)$, respectively. These functions were introduced by considering the extended beta function (7) instead of beta function (1) in the general term of series (4) and (5). They defined these functions as

$$
\begin{gathered}
F_{\sigma}(a, b ; c ; z)=\sum_{n=0}^{\infty} \frac{(a)_{n} B(b+n, c-b ; \sigma)}{B(b, c-b)} \frac{z^{n}}{n !}, \\
\sigma \geq 0, \quad|z|<1, \quad \operatorname{Re}(c)>\operatorname{Re}(b)>0, \\
\Phi_{\sigma}(b ; c ; z)=\sum_{n=0}^{\infty} \frac{B(b+n, c-b ; \sigma)}{B(b, c-b)} \frac{z^{n}}{n !}, \\
\sigma \geq 0, \quad \operatorname{Re}(c)>\operatorname{Re}(b)>0 .
\end{gathered}
$$

Using the integral representation of the extended beta function (7) in (8) and (9), the integral representations of extended hypergeometric functions, for $\operatorname{Re}(c)>\operatorname{Re}(b)>0$ and $\sigma \geq 0$, are obtained as

$$
\begin{aligned}
F_{\sigma}(a, b ; c ; z)= & \frac{1}{B(b, c-b)} \\
& \times \int_{0}^{1} \frac{t^{b-1}(1-t)^{c-b-1}}{(1-z t)^{a}} \exp \left[-\frac{\sigma}{t(1-t)}\right] d t, \\
\Phi_{\sigma}(b ; c ; z)= & \frac{1}{B(b, c-b)} \quad(10) \\
& \times \int_{0}^{1} t^{b-1}(1-t)^{c-b-1} \exp \left[z t-\frac{\sigma}{t(1-t)}\right] d t .
\end{aligned}
$$

Substituting $\sigma=0$ in (8) or $(10)$, we have $F_{0}(a, b ; c ; z)=$ $F(a, b ; c ; z)$; that is, the classical Gauss hypergeometric function is a special case of the extended Gauss hypergeometric function. Similarly, by taking $\sigma=0$ in (9) or (11), we have $\Phi_{0}(b ; c ; z)=\Phi(b ; c ; z)$, which means that the classical confluent hypergeometric function is a special case of the extended confluent hypergeometric function. Chaudhry et al. [5] found that the extended hypergeometric functions are related to the extended beta, Bessel, and Whittaker functions and also gave several alternative integral representations.

The classical functions, such as gamma, beta, confluent hypergeometric, Gauss hypergeometric, Bessel, and Whittaker, have been generalized to the matrix case and their properties have been studied extensively. For example, see
Butler and Wood [6-8], Herz [9], Constantine [10], James [11], Muirhead [12], and Gupta and Nagar [13]. Many distributions of random matrices and their functions such as determinant and trace and moments of test statistics can be expressed in terms of hypergeometric functions of matrix arguments. For some recent work, the reader is referred to Bekker et al. [14, 15], Bekker et al. [16], and Gupta and Nagar [17]. Recently, Nagar et al. [18] have defined and studied the extended beta function of matrix argument.

The extended Gauss hypergeometric function and extended confluent hypergeometric function have not been generalized to the matrix case and therefore the main objective of this work is to define these generalizations, give various integral representations, study their properties, and establish their relationships with other special functions of matrix argument.

This paper is divided into eight sections. Section 2 deals with some well-known definitions and results on matrix algebra, multivariate gamma function, multivariate beta function, and special functions. In Section 3, the extended Gauss hypergeometric function of matrix argument has been defined and its properties have been studied. Section 4 deals with the extended confluent hypergeometric function of matrix argument and Section 5 defines the extended Whittaker function of matrix argument. Section 6 is devoted to several integrals involving these newly defined functions. The results contained in this section show the relationship of these functions with some known special functions. Finally, Sections 7 and 8 give a number of matrix variate distributions.

\section{Some Known Definitions and Results}

This section provides definitions and important properties of some classical special functions that are critical to the development of this work.

Replacing the confluent hypergeometric function that appears in (6) by its integral representation (3), we obtain the integral representation of the Whittaker function as

$$
\begin{aligned}
M_{k, \mu}(z)= & \frac{z^{\mu+1 / 2} \exp (-z / 2)}{B(\mu-k+1 / 2, \mu+k+1 / 2)} \\
& \times \int_{0}^{1} t^{\mu-k-1 / 2}(1-t)^{\mu+k-1 / 2} \exp (z t) d t .
\end{aligned}
$$

Another integral representation of $M_{k, \mu}(z)$ is obtained by substituting $u=2 t-1$ in (12), to get

$$
\begin{aligned}
M_{k, \mu}(z)= & \frac{2^{-2 \mu} z^{\mu+1 / 2}}{B(\mu-k+1 / 2, \mu+k+1 / 2)} \\
& \times \int_{-1}^{1}(1+u)^{\mu-k-1 / 2}(1-u)^{\mu+k-1 / 2} \exp \left(\frac{z u}{2}\right) d u .
\end{aligned}
$$

Further, application of Kummer's transformation, namely,

$$
\Phi(b ; c ; z)=\exp (z) \Phi(c-b ; c ;-z),
$$


in (6) yields

$$
M_{k, \mu}(-z)=(-1)^{\mu+1 / 2} M_{-k, \mu}(z) .
$$

Let $A=\left(a_{i j}\right)$ be an $m \times m$ matrix of real or complex numbers. Then, $A^{\prime}$ denotes the transpose of $A ; \operatorname{tr}(A)=$ $a_{11}+\cdots+a_{m m} ; \operatorname{etr}(A)=\exp (\operatorname{tr}(A)) ; \operatorname{det}(A)=$ determinant of $A ; A=A^{\prime} \geq 0$ means that $A$ is symmetric positive semidefinite; $A=A^{\prime}>0$ means that $A$ is symmetric positive definite, $0<A<I_{m}$ means that both $A$ and $I_{m}-A$ are symmetric positive definite, and $A^{1 / 2}$ denotes the unique positive definite square root of $A>0$.

Several generalizations of Euler's gamma function are available in the scientific literature. The multivariate gamma function which is frequently used in multivariate statistical analysis is defined by (Ingham [19] and Siegel [20])

$$
\Gamma_{m}(a)=\int_{X>0} \operatorname{etr}(-X) \operatorname{det}(X)^{a-(m+1) / 2} d X,
$$

where $\operatorname{Re}(a)>(m-1) / 2$ and the integration is carried out over $m \times m$ symmetric positive definite matrices. By evaluating the above integral, it is easy to see that

$$
\begin{array}{r}
\Gamma_{m}(a)=\pi^{m(m-1) / 4} \prod_{j=1}^{m} \Gamma\left(a-\frac{j-1}{2}\right), \\
\operatorname{Re}(a)>\frac{m-1}{2} .
\end{array}
$$

Let $A$ be an $m \times m$ symmetric positive definite matrix and make the transformation $X=A^{1 / 2} Y A^{1 / 2}$, where $A^{1 / 2}$ is the positive definite square root of $A$, with the Jacobian $J(X \rightarrow$ $Y)=\operatorname{det}(A)^{(m+1) / 2}$. Then,

$$
\begin{gathered}
\int_{Y>0} \operatorname{etr}(-A Y) \operatorname{det}(Y)^{a-(m+1) / 2} d Y \\
=\operatorname{det}(A)^{-a} \Gamma_{m}(a) .
\end{gathered}
$$

The above result also holds for complex symmetric $A$ with $\operatorname{Re}(A)>0$ by analytic continuation. The multivariate generalization of the beta function is given by

$$
\begin{aligned}
B_{m} & (a, b) \\
& =\int_{0<X<I_{m}} \operatorname{det}(X)^{a-(m+1) / 2} \operatorname{det}\left(I_{m}-X\right)^{b-(m+1) / 2} d X \\
& =\frac{\Gamma_{m}(a) \Gamma_{m}(b)}{\Gamma_{m}(a+b)}=B_{m}(b, a),
\end{aligned}
$$

where $\operatorname{Re}(a)>(m-1) / 2$ and $\operatorname{Re}(b)>(m-1) / 2$.

Siegel [20] established the identity

$$
B_{m}(a, b)=\int_{Y>0} \frac{\operatorname{det}(Y)^{a-(m+1) / 2}}{\operatorname{det}\left(I_{m}+Y\right)^{a+b}} d Y
$$

which can be derived from (19) by using the matrix transformation $X=\left(I_{m}+Y\right)^{-1} Y$ with the Jacobian $J(X \rightarrow Y)=$ $\operatorname{det}\left(I_{m}+Y\right)^{-(m+1)}$.

The type 3 Bessel function of Herz (Herz [9, p. 517, p. $506]), K_{\delta}(Z)$, of $m \times m$ symmetric positive definite matrix argument $Z$ is defined by

$$
\begin{aligned}
& K_{\delta}(Z) \\
& \quad=2^{-m} \int_{R>0} \operatorname{det}(R)^{\delta-(m+1) / 2} \operatorname{etr}\left[-\frac{1}{2}\left(R+R^{-1}\right) Z\right] d R .
\end{aligned}
$$

The Gauss hypergeometric function of $m \times m$ symmetric matrix argument $X$, denoted by $F(a, b ; c ; X)$, is defined by

$$
\begin{aligned}
& F(a, b ; c ; X) \\
& =\frac{1}{B_{m}(b, c-b)} \\
& \quad \times \int_{0<Z<I_{m}} \frac{\operatorname{det}(Z)^{b-(m+1) / 2} \operatorname{det}\left(I_{m}-Z\right)^{c-b-(m+1) / 2}}{\operatorname{det}\left(I_{m}-X Z\right)^{a}} d Z,
\end{aligned}
$$

where $X<I_{m}, \operatorname{Re}(b)>(m-1) / 2$, and $\operatorname{Re}(c-b)>(m-1) / 2$. The confluent hypergeometric function of $m \times m$ symmetric matrix argument $X$, denoted by $\Phi(b ; c ; X)$, is defined by

$$
\begin{aligned}
\Phi(b ; c ; X)= & \frac{1}{B_{m}(b, c-b)} \\
& \quad \times \int_{0<Z<I_{m}} \operatorname{det}(Z)^{b-(m+1) / 2} \\
& \quad \times \operatorname{det}\left(I_{m}-Z\right)^{c-b-(m+1) / 2} \operatorname{etr}(X Z) d Z,
\end{aligned}
$$

where $\operatorname{Re}(b)>(m-1) / 2$ and $\operatorname{Re}(c-b)>(m-1) / 2$. If we make the transformation $Z=\left(I_{m}+U\right)^{-1} U$ in (22) and (23) with the Jacobian $J(Z \rightarrow U)=\operatorname{det}\left(I_{m}+U\right)^{-(m+1)}$, we obtain alternative integral representations for $F(a, b ; c ; X)$ and $\Phi(b ; c ; X)$ as

$$
\begin{aligned}
& F(a, b ; c ; X) \\
& =\frac{1}{B_{m}(b, c-b)} \\
& \quad \times \int_{U>0} \frac{\operatorname{det}(U)^{b-(m+1) / 2} \operatorname{det}\left(I_{m}+U\right)^{a-c}}{\operatorname{det}\left(I_{m}+\left(I_{m}-X\right) U\right)^{a}} d U, \\
& \Phi(b ; c ; X) \\
& =\frac{1}{B_{m}(b, c-b)} \\
& \quad \times \int_{U>0} \frac{\operatorname{det}(U)^{b-(m+1) / 2} \operatorname{etr}\left[X\left(I_{m}+U\right)^{-1} U\right]}{\operatorname{det}\left(I_{m}+U\right)^{c}} d U .
\end{aligned}
$$


Further, substituting $Y=\left(I_{m}-X\right)^{1 / 2} U\left(I_{m}-X\right)^{1 / 2}$ with the Jacobian $J(U \rightarrow Y)=\operatorname{det}\left(I_{m}-X\right)^{-(m+1) / 2}$ in (24), we get another interesting integral form of $F(a, b ; c ; X)$ as

$$
\begin{aligned}
& F(a, b ; c ; X) \\
& =\frac{\operatorname{det}\left(I_{m}-X\right)^{-b}}{B_{m}(b, c-b)} \\
& \quad \times \int_{Y>0} \frac{\operatorname{det}(Y)^{b-(m+1) / 2} \operatorname{det}\left(I_{m}+\left(I_{m}-X\right)^{-1} Y\right)^{a-c}}{\operatorname{det}\left(I_{m}+Y\right)^{a}} d Y .
\end{aligned}
$$

Putting $X=I_{m}$ in (22) and evaluating the resulting integral using (19), one obtains

$$
F\left(a, b ; c ; I_{m}\right)=\frac{\Gamma_{m}(c) \Gamma_{m}(c-a-b)}{\Gamma_{m}(c-a) \Gamma_{m}(c-b)},
$$

where $\operatorname{Re}(c-a-b)>(m-1) / 2$. Putting $a=c$ in (26) and using (20), one can easily show that

$$
F(c, b ; c ; X)=\operatorname{det}\left(I_{m}-X\right)^{-b} .
$$

Transforming $Z \rightarrow I_{m}-Z$, (23) becomes

$$
\begin{aligned}
\Phi(b ; c ; X)= & \frac{\operatorname{etr}(X)}{B_{m}(b, c-b)} \\
& \quad \times \int_{0<Z<I_{m}} \operatorname{det}\left(I_{m}-Z\right)^{b-(m+1) / 2} \\
& \quad \times \operatorname{det}(Z)^{c-b-(m+1) / 2} \operatorname{etr}(-X Z) d Z .
\end{aligned}
$$

A comparison of (23) and (29) leads to the well-known Kummer's relation (Herz [9, Eq. 2.8, p. 488]):

$$
\Phi(b ; c ; X)=\operatorname{etr}(X) \Phi(c-b ; c ;-X) .
$$

Further, by using the transformation $2 Z \rightarrow Z+I_{m}$, (23) can be written as

$$
\begin{aligned}
\Phi(b ; c ; X)= & \frac{2^{-m c+m(m+1) / 2} \operatorname{etr}(X / 2)}{B_{m}(b, c-b)} \\
& \times \int_{-I_{m}<Z<I_{m}} \operatorname{det}\left(I_{m}-Z\right)^{c-b-(m+1) / 2} \\
& \quad \times \operatorname{det}\left(I_{m}+Z\right)^{b-(m+1) / 2} \operatorname{etr}\left(\frac{X Z}{2}\right) d Z .
\end{aligned}
$$

For properties and further results on these functions, the reader is referred to Constantine [10], James [11], Muirhead [12], and Gupta and Nagar [13]. The numerical computation of a hypergeometric function of matrix arguments is very difficult. However, some numerical methods are proposed in recent years; see Hashiguchi et al. [21] and Koev and Edelman [22].
Also, in 1968, Abdi [23] defined the Whittaker function of matrix argument expressing it in terms of a confluent hypergeometric function of matrix argument $\Phi(b ; c ; X)$ as

$$
\begin{aligned}
M(k, \mu ; X)= & \operatorname{det}(X)^{\mu+(m+1) / 4} \operatorname{etr}\left(-\frac{X}{2}\right) \\
& \times \Phi\left(\mu-k+\frac{m+1}{4} ; 2 \mu+\frac{m+1}{2} ; X\right),
\end{aligned}
$$

where $\operatorname{Re}(\mu \pm k)>(m-3) / 4$. He also studied several properties and integral representations of this function. It is apparent that, by using different integral representations of $\Phi$ in (32), a variety of integral representations for $M(k, \mu ; X)$ can be obtained. For example, using (31) in (32), we get

$$
\begin{aligned}
& M(k, \mu ; X) \\
& =\frac{2^{-2 m \mu} \operatorname{det}(X)^{\mu+(m+1) / 4}}{B_{m}(\mu+k+(m+1) / 4, \mu-k+(m+1) / 4)} \\
& \quad \times \int_{-I_{m}<Z<I_{m}} \operatorname{det}\left(I_{m}+Z\right)^{\mu-k-(m+1) / 4} \\
& \quad \times \operatorname{det}\left(I_{m}-Z\right)^{\mu+k-(m+1) / 4} \operatorname{etr}\left(\frac{X Z}{2}\right) d Z .
\end{aligned}
$$

Next, we give definition and properties of the extended beta function of matrix argument due to Nagar et al. [18].

Definition 1. The extended matrix variate beta function, denoted by $B_{m}(b, c ; \Sigma)$, is defined as

$$
\begin{aligned}
B_{m}(b, c ; \Sigma)=\int_{0<Z<I_{m}} & \operatorname{etr}\left[-\Sigma Z^{-1}\left(I_{m}-Z\right)^{-1}\right] \\
& \times \operatorname{det}(Z)^{b-(m+1) / 2} \\
& \times \operatorname{det}\left(I_{m}-Z\right)^{c-(m+1) / 2} d Z,
\end{aligned}
$$

where $b$ and $c$ are arbitrary complex numbers and $\operatorname{Re}(\Sigma)>0$.

From the definition, it is apparent that the function $B_{m}(b$, $c ; \Sigma)$ is invariant under the transformation $Z \rightarrow H Z H^{\prime}$, $H \in O(m)$; thereby, $B_{m}(b, c ; \Sigma)$ is a function of the eigenvalues of the matrix $\Sigma$. If we take $\Sigma=0$ in (34), then for $\operatorname{Re}(b)>$ $(m-1) / 2$ and $\operatorname{Re}(c)>(m-1) / 2$ we have $B_{m}(b, c ; 0)=B_{m}(b, c)$. Further, replacing $Z$ by $I_{m}-Z$ in (34), one can show that $B_{m}(b, c ; \Sigma)=B_{m}(c, b ; \Sigma)$.

Now, applying the transformation $Z=\left(I_{m}+U\right)^{-1} U$ in (34) with the Jacobian $J(Z \rightarrow U)=\operatorname{det}\left(I_{m}+U\right)^{-(m+1)}$, we arrive at

$$
\begin{aligned}
& B_{m}(b, c ; \Sigma) \\
&= \operatorname{etr}(-2 \Sigma) \\
& \quad \times \int_{U>0} \frac{\operatorname{det}(U)^{b-(m+1) / 2} \operatorname{etr}\left[-\Sigma\left(U+U^{-1}\right)\right]}{\operatorname{det}\left(I_{m}+U\right)^{b+c}} d U .
\end{aligned}
$$

If we take $b=-c$ in (35) and compare the resulting expression with (21), we obtain an interesting relation between the 
extended matrix variate beta function and the type 3 Bessel function of Herz as

$$
B_{m}(b,-b ; \Sigma)=2^{m} \operatorname{etr}(-2 \Sigma) K_{b}(2 \Sigma), \quad \Sigma>0 .
$$

Also, from (20) and (35), one can prove the inequality

$$
B_{m}(b, c ; \Sigma) \leq \operatorname{etr}(-4 \Sigma) B_{m}(b, c) \leq \frac{\exp (-m)}{\operatorname{det}(4 \Sigma)} B_{m}(b, c)
$$

Let $f(X)$ be a scalar valued function of an $m \times m$ symmetric positive definite matrix $X$ such that $f(X)=$ $f\left(H X H^{\prime}\right), H \in O(m)$. Then, the $M$-transform of $f(X)$, denoted by $M_{s}(f)$, is defined by

$$
M_{s}(f)=\int_{X>0} \operatorname{det}(\Sigma)^{s-(m+1) / 2} f(X) d X,
$$

where $\operatorname{Re}(s)>(m-1) / 2$.

The $M$-transform of the extended beta function of the matrix argument is given by

$$
\begin{gathered}
\int_{\Sigma>0} \operatorname{det}(\Sigma)^{s-(m+1) / 2} B_{m}(b, c ; \Sigma) d \Sigma \\
=\Gamma_{m}(s) B_{m}(b+s, c+s)
\end{gathered}
$$

where $\operatorname{Re}(s)>(m-1) / 2, \operatorname{Re}(b+s)>(m-1) / 2$, and $\operatorname{Re}(c+s)>$ $(m-1) / 2$.

\section{Extended Gauss Hypergeometric Function of Matrix Argument}

In this section, we define the extended Gauss hypergeometric function of matrix argument (EGHFMA), which is a matrix variate generalization of the extended Gauss hypergeometric function (10) and an extended form of the classical Gauss hypergeometric function of matrix argument defined in (22). We also give several integral representations and properties of this function.

Definition 2. The extended Gauss hypergeometric function of matrix argument (EGHFMA), denoted by $F_{\Sigma}(a, b ; c ; X)$, is defined for an $m \times m$ symmetric matrix $X$ as

$$
\begin{aligned}
& F_{\Sigma}(a, b ; c ; X) \\
& =\frac{1}{B_{m}(b, c-b)} \\
& \quad \times \int_{0<Z<I_{m}} \operatorname{etr}\left[-\Sigma Z^{-1}\left(I_{m}-Z\right)^{-1}\right] \\
& \quad \times \frac{\operatorname{det}(Z)^{b-(m+1) / 2} \operatorname{det}\left(I_{m}-Z\right)^{c-b-(m+1) / 2}}{\operatorname{det}\left(I_{m}-X Z\right)^{a}} d Z,
\end{aligned}
$$

where $X<I_{m}, \Sigma \geq 0, \operatorname{Re}(b)>(m-1) / 2$, and $\operatorname{Re}(c-b)>$ $(m-1) / 2$.

If we take $\Sigma=0$ in (40), then EGHFMA reduces to a classical Gauss hypergeometric function of matrix argument
(22); that is, $F_{0}(a, b ; c ; X)=F(a, b ; c ; X)$. Also, if we consider $X=I_{m}$ in (40) and compare the resulting expression with representation (34), we find that the extended beta function of matrix argument and EGHFMA are connected by the expression

$$
F_{\Sigma}\left(a, b ; c ; I_{m}\right)=\frac{B_{m}(b, c-b-a ; \Sigma)}{B_{m}(b, c-b)} .
$$

Further, substituting $c=a$ in (41) and using (36), we obtain

$$
F_{\Sigma}\left(a, b ; a ; I_{m}\right)=\frac{B_{m}(b,-b ; \Sigma)}{B_{m}(b, a-b)}=\frac{2^{m} \operatorname{etr}(-2 \Sigma)}{B_{m}(b, a-b)} K_{b}(2 \Sigma) \text {. }
$$

Theorem 3. For $\Sigma \geq 0, H \in O(m)$, and $\lambda<1$, we have $F_{H \Sigma H^{\prime}}\left(a, b ; c ; \lambda I_{m}\right)=F_{\Sigma}\left(a, b ; c ; \lambda I_{m}\right)$. That is, $F_{\Sigma}\left(a, b ; c ; \lambda I_{m}\right)$, $\lambda<1$, is a function of the eigenvalues of the matrix $\sum$. Further, for $H \in O(m), F_{\sigma I_{m}}\left(a, b ; c ; H X H^{\prime}\right)=F_{\sigma I_{m}}(a, b ; c ; X)$ which indicates that $F_{\sigma I_{m}}(a, b ; c ; X), \sigma \geq 0$, is a function of the eigenvalues of the matrix $X$.

Proof. Substituting $X=\lambda I_{m}$ with $\lambda<1$ and replacing $\Sigma$ by $H \Sigma H^{\prime}, H \in O(m)$, in (40), we arrive at

$$
\begin{aligned}
F_{H \Sigma H^{\prime}}\left(a, b ; c ; \lambda I_{m}\right) \\
=\frac{1}{B_{m}(b, c-b)} \\
\quad \times \int_{0<Z<I_{m}} \frac{\operatorname{det}(Z)^{b-(m+1) / 2} \operatorname{det}\left(I_{m}-Z\right)^{c-b-(m+1) / 2}}{\operatorname{det}\left(I_{m}-\lambda Z\right)^{a}} \\
\quad \quad \times \operatorname{etr}\left[-H \Sigma H^{\prime} Z^{-1}\left(I_{m}-Z\right)^{-1}\right] d Z \\
=F_{\Sigma}\left(a, b ; c ; \lambda I_{m}\right),
\end{aligned}
$$

where the last line has been obtained by substituting $Z=$ $H Y H^{\prime}$ with the Jacobian $J(Z \rightarrow Y)=1$ and using (40). This means that $F_{\Sigma}\left(a, b, c, \lambda I_{m}\right), \lambda<1$, is a function of the eigenvalues of the matrix $\Sigma$. Similarly, if in (40) we take $\Sigma=$ $\sigma I_{m}$ with $\sigma \geq 0$ and $X$ is replaced by $H X H^{\prime}, H \in O(m)$, we obtain

$$
F_{\sigma I_{m}}\left(a, b ; c ; H X H^{\prime}\right)=F_{\sigma I_{m}}(a, b ; c ; X),
$$

which shows that $F_{\sigma I_{m}}(a, b ; c ; X), \sigma \geq 0$, is a function of the eigenvalues of the matrix $X$.

The following theorem gives an extended form of the integral representation given in (24).

Theorem 4. Let $X$ be an $m \times m$ symmetric matrix such that $X<I_{m}, \Sigma \geq 0, \operatorname{Re}(b)>(m-1) / 2$, and $\operatorname{Re}(c-b)>(m-1) / 2$. Then,

$$
\begin{aligned}
F_{\Sigma}(a, b ; c ; X) & \\
= & \frac{\operatorname{etr}(-2 \Sigma)}{B_{m}(b, c-b)} \\
& \quad \times \int_{U>0} \frac{\operatorname{det}(U)^{b-(m+1) / 2} \operatorname{etr}\left[-\Sigma\left(U+U^{-1}\right)\right]}{\operatorname{det}\left(I_{m}+U\right)^{c-a} \operatorname{det}\left(I_{m}+\left(I_{m}-X\right) U\right)^{a}} d U .
\end{aligned}
$$


Proof. In the integral representation of EGHFMA given in (40), substituting $Z=\left(I_{m}+U\right)^{-1} U$ with the Jacobian $J(Z \rightarrow$ $U)=\operatorname{det}\left(I_{m}+U\right)^{-(m+1)}$, we obtain the desired result.

Theorem 5. If $X$ is an $m \times m$ symmetric matrix such that $X<$ $I_{m}, \Sigma>0, b>(m-1) / 2$, and $c-b>(m-1) / 2$, then

$$
\begin{aligned}
F_{\Sigma}(a, b ; c ; X) & \leq \operatorname{etr}(-4 \Sigma) F(a, b ; c ; X) \\
& \leq \frac{\exp (-m)}{\operatorname{det}(4 \Sigma)} F(a, b ; c ; X) .
\end{aligned}
$$

Proof. From the Trace Inequality given in Abadir and Magnus $\left[24\right.$, p. 338], it follows that $\operatorname{tr}\left[\Sigma\left(U+U^{-1}-2 I_{m}\right)\right] \geq 0$ which implies that $\operatorname{tr}\left[\Sigma\left(U+U^{-1}\right)\right] \geq 2 \operatorname{tr}(\Sigma)$ and

$$
\operatorname{etr}\left[-\Sigma\left(U+U^{-1}\right)\right] \leq \operatorname{etr}(-2 \Sigma), \quad U>0, \quad \Sigma>0 .
$$

Now, using the above inequality in the integral given in (45), we get

$$
\begin{aligned}
F_{\Sigma}(a, b ; c ; X) \leq & \frac{\operatorname{etr}(-4 \Sigma)}{B_{m}(b, c-b)} \\
& \times \int_{U>0} \frac{\operatorname{det}(U)^{b-(m+1) / 2} \operatorname{det}\left(I_{m}+U\right)^{a-c}}{\operatorname{det}\left(I_{m}+\left(I_{m}-X\right) U\right)^{a}} d U \\
= & \operatorname{etr}(-4 \Sigma) F(a, b ; c ; X),
\end{aligned}
$$

where the last line has been obtained by using (24). If $A$ is an $m \times m$ positive definite matrix, then it has been shown in Abadir and Magnus [24, p. 333] that $\ln \operatorname{det}(A) \leq \operatorname{tr}(A)-m$. This inequality, for $A=4 \Sigma$, yields

$$
\operatorname{etr}(-4 \Sigma) \leq \frac{\exp (-m)}{\operatorname{det}(4 \Sigma)}
$$

which gives the second part of the inequality.

If we take $X=I_{m}$ in (46) and then use (41) and (27) in the resulting expression, we obtain

$$
\begin{aligned}
B_{m}(b, d ; \Sigma) & \leq \operatorname{etr}(-4 \Sigma) B_{m}(b, d) \\
& \leq \frac{\exp (-m)}{\operatorname{det}(4 \Sigma)} B_{m}(b, d),
\end{aligned}
$$

where $d=c-a-b>(m-1) / 2$.

The following theorem gives $M$-transform of the extended matrix variate Gauss hypergeometric function $F_{\Sigma}(a, b$; $c ; X)$.

Theorem 6. If $X$ is an $m \times m$ symmetric matrix such that $X<$ $I_{m}, \operatorname{Re}(s)>(m-1) / 2, \operatorname{Re}(b)>(m-1) / 2$, and $\operatorname{Re}(c-b)>$ $(m-1) / 2$, then

$$
\begin{aligned}
\int_{\Sigma>0} & \operatorname{det}(\Sigma)^{s-(m+1) / 2} F_{\Sigma}(a, b ; c ; X) d \Sigma \\
= & \frac{\Gamma_{m}(s) B_{m}(b+s, c-b+s)}{B_{m}(b, c-b)} \\
& \times F(a, b+s ; c+2 s ; X) .
\end{aligned}
$$

Proof. Replacing $F_{\Sigma}(a, b ; c ; X)$ by its integral representation given in (40) and changing the order of integration, we get

$$
\begin{aligned}
\int_{\Sigma>0} & \operatorname{det}(\Sigma)^{s-(m+1) / 2} F_{\Sigma}(a, b ; c ; X) d \Sigma \\
= & \frac{1}{B_{m}(b, c-b)} \\
& \times \int_{0<Z<I_{m}} \frac{\operatorname{det}(Z)^{b-(m+1) / 2} \operatorname{det}\left(I_{m}-Z\right)^{c-b-(m+1) / 2}}{\operatorname{det}\left(I_{m}-X Z\right)^{a}} \\
& \times \int_{\Sigma>0} \operatorname{det}(\Sigma)^{s-(m+1) / 2} \operatorname{etr}\left[-\Sigma Z^{-1}\left(I_{m}-Z\right)^{-1}\right] d \Sigma d Z .
\end{aligned}
$$

Now, using (18), we arrive at

$$
\begin{aligned}
\int_{\Sigma>0} & \operatorname{det}(\Sigma)^{s-(m+1) / 2} F_{\Sigma}(a, b ; c ; X) d \Sigma \\
= & \frac{\Gamma_{m}(s)}{B_{m}(b, c-b)} \\
& \times \int_{0<Z<I_{m}} \frac{\operatorname{det}(Z)^{b+s-(m+1) / 2} \operatorname{det}\left(I_{m}-Z\right)^{c-b+s-(m+1) / 2} d Z}{\operatorname{det}\left(I_{m}-X Z\right)^{a}} .
\end{aligned}
$$

Finally, the last integral is replaced by the Gauss hypergeometric function of matrix argument by using representation (22).

Substitution of $s=(m+1) / 2$ in (51) gives the following interesting relationship between EGHFMA and classical Gauss hypergeometric function of matrix argument:

$$
\begin{aligned}
\int_{\Sigma>0} & F_{\Sigma}(a, b ; c ; X) d \Sigma \\
= & \frac{\Gamma_{m}[(m+1) / 2] B_{m}(b+(m+1) / 2, c-b+(m+1) / 2)}{B_{m}(b, c-b)} \\
& \times F\left(a, b+\frac{m+1}{2} ; c+m+1 ; X\right) .
\end{aligned}
$$

Also, if we take $X=I_{m}$ in (51) and then use (41) and (27) in the resulting expression, we obtain the $M$-transform of the extended beta function of matrix argument as

$$
\begin{gathered}
\int_{\Sigma>0} \operatorname{det}(\Sigma)^{s-(m+1) / 2} B_{m}(b, d ; \Sigma) d \Sigma \\
=\frac{\Gamma_{m}(s) \Gamma_{m}(d+s) \Gamma_{m}(b+s)}{\Gamma_{m}(b+d+2 s)},
\end{gathered}
$$

where $d=c-a-b$ with $\operatorname{Re}(d)>(m-1) / 2$.

The transformation formula for the extended Gauss hypergeometric function of matrix argument is given next. 
Theorem 7. If $X$ is an $m \times m$ symmetric matrix such that $X<$ $I_{m}, \Sigma \geq 0, \operatorname{Re}(b)>(m-1) / 2$, and $\operatorname{Re}(c-b)>(m-1) / 2$, then

$$
\begin{aligned}
F_{\Sigma}(a, b ; c ; X)= & \operatorname{det}\left(I_{m}-X\right)^{-a} \\
& \times F_{\Sigma}\left(a, c-b ; c ;-X\left(I_{m}-X\right)^{-1}\right) .
\end{aligned}
$$

Proof. Making the transformation $Z \rightarrow I_{m}-Z$ in the integral representation given in (40), one obtains

$$
\begin{aligned}
& F_{\Sigma}(a, b ; c ; X) \\
& =\frac{1}{B_{m}(b, c-b)} \\
& \quad \times \int_{0<Z<I_{m}} \frac{\operatorname{det}\left(I_{m}-Z\right)^{b-(m+1) / 2} \operatorname{det}(Z)^{c-b-(m+1) / 2}}{\operatorname{det}\left(I_{m}-X\left(I_{m}-Z\right)\right)^{a}} \\
& \quad \times \operatorname{etr}\left[-\Sigma Z^{-1}\left(I_{m}-Z\right)^{-1}\right] d Z .
\end{aligned}
$$

Now, writing

$$
\begin{aligned}
& \operatorname{det}\left(I_{m}-X\left(I_{m}-Z\right)\right)^{-a} \\
& \quad=\operatorname{det}\left(I_{m}-X\right)^{-a} \operatorname{det}\left(I_{m}+\left(I_{m}-X\right)^{-1} X Z\right)^{-a}
\end{aligned}
$$

in (57) and noting that $B_{m}(b, c-b)=B_{m}(c-b, b)$, we have

$$
\begin{aligned}
& F_{\Sigma}(a, b ; c ; X) \\
& =\frac{\operatorname{det}\left(I_{m}-X\right)^{-a}}{B_{m}(c-b, b)} \\
& \quad \times \int_{0<Z<I_{m}} \frac{\operatorname{det}\left(I_{m}-Z\right)^{b-(m+1) / 2} \operatorname{det}(Z)^{c-b-(m+1) / 2}}{\operatorname{det}\left(I_{m}+\left(I_{m}-X\right)^{-1} X Z\right)^{a}} \\
& \quad \times \operatorname{etr}\left[-\sum Z^{-1}\left(I_{m}-Z\right)^{-1}\right] d Z .
\end{aligned}
$$

Finally, evaluating the above integral by using (40), we get the desired result.

It is noteworthy that $\Sigma=0$ in (56) gives the well-known transformation formula

$$
\begin{aligned}
F(a, b ; c ; X)= & \operatorname{det}\left(I_{m}-X\right)^{-a} \\
& \times F\left(a, c-b ; c ;-X\left(I_{m}-X\right)^{-1}\right) .
\end{aligned}
$$

\section{Extended Confluent Hypergeometric Function of Matrix Argument}

In this section, we define and study the extended confluent hypergeometric function of matrix argument (ECHFMA), which is a generalization to the matrix case of the extended confluent hypergeometric function $\Phi_{\sigma}(b ; c ; z)$.
Definition 8 . The extended confluent hypergeometric function of an $m \times m$ symmetric matrix argument (ECHFMA), denoted by $\Phi_{\Sigma}(b ; c ; X)$, is defined as

$$
\begin{aligned}
& \Phi_{\Sigma}(b ; c ; X) \\
& =\frac{1}{B_{m}(b, c-b)} \\
& \quad \times \int_{0<Z<I_{m}} \operatorname{etr}\left[X Z-\Sigma Z^{-1}\left(I_{m}-Z\right)^{-1}\right] \\
& \quad \quad \times \operatorname{det}(Z)^{b-(m+1) / 2} \operatorname{det}\left(I_{m}-Z\right)^{c-b-(m+1) / 2} d Z,
\end{aligned}
$$

where $\operatorname{Re}(b)>(m-1) / 2, \operatorname{Re}(c-b)>(m-1) / 2$, and $\Sigma \geq 0$.

If we take $\Sigma=0$ in (61), then ECHFMA becomes the confluent hypergeometric function of matrix argument; that is, $\Phi_{0}(b ; c ; X)=\Phi(b ; c ; X)$. Also, if we put $X=0$ in (61) and compare the resulting expression with (34), we will arrive at the conclusion that the ECHFMA and extended beta function of matrix argument retain the relationship

$$
\Phi_{\Sigma}(b ; c ; 0)=\frac{B_{m}(b, c-b ; \Sigma)}{B_{m}(b, c-b)} .
$$

Theorem 9. If $\Sigma>0$ and $H \in O(m)$, then $\Phi_{H \Sigma H^{\prime}}\left(b ; c ; \lambda I_{m}\right)=$ $\Phi_{\Sigma}\left(b ; c ; \lambda I_{m}\right), \lambda \in \mathbb{R}$. That is, $\Phi_{\Sigma}\left(b ; c ; \lambda I_{m}\right)$ is a function of the eigenvalues of the matrix $\Sigma$. Further, for $H \in$ $O(m), \Phi_{\sigma I_{m}}\left(b ; c ; H X H^{\prime}\right)=\Phi_{\sigma I_{m}}(b ; c ; X)$ which indicates that $\Phi_{\sigma I_{m}}(b ; c ; X), \sigma \geq 0$, is a function of the eigenvalues of the matrix $X$.

Proof. The proof is similar to the proof of Theorem 3.

Theorem 10. Let $X$ and $\Sigma$ be $m \times m$ symmetric matrices with $\Sigma \geq 0$. If $\operatorname{Re}(b)>(m-1) / 2$ and $\operatorname{Re}(c-b)>(m-1) / 2$, then

$$
\begin{aligned}
& \Phi_{\Sigma}(b ; c ; X) \\
& =\frac{\operatorname{etr}(X)}{B_{m}(b, c-b)} \\
& \quad \times \int_{0<U<I_{m}} \operatorname{etr}\left[-X U-\Sigma U^{-1}\left(I_{m}-U\right)^{-1}\right] \\
& \quad \quad \times \operatorname{det}(U)^{c-b-(m+1) / 2} \operatorname{det}\left(I_{m}-U\right)^{b-(m+1) / 2} d U .
\end{aligned}
$$

Proof. In the integral representation of the ECHFMA given in (61), consider the substitution $I_{m}-U=Z$ with the Jacobian $J(Z \rightarrow U)=1$.

Corollary 11. Let $X$ and $\Sigma$ be $m \times m$ symmetric matrices with $\Sigma \geq 0$. If $\operatorname{Re}(b)>(m-1) / 2$ and $\operatorname{Re}(c-b)>(m-1) / 2$, then

$$
\Phi_{\Sigma}(b ; c ; X)=\operatorname{etr}(X) \Phi_{\Sigma}(c-b ; c ;-X) .
$$

Proof. The desired result is obtained by evaluating the integral in (63) by using (61). 
For $\Sigma=0$, expression (64) reduces to the well-known Kummer's relation for the classical confluent hypergeometric function of matrix argument. Moreover, the previous corollary is the generalization to the matrix case of Kummer's relation for the extended confluent hypergeometric function of scalar argument.

Theorem 12. If $\operatorname{Re}(b)>(m-1) / 2$ and $\operatorname{Re}(c-b)>(m-1) / 2$, then

$$
\begin{aligned}
& \Phi_{\Sigma}(b ; c ; X) \\
& =\frac{\operatorname{etr}(-2 \Sigma)}{B_{m}(b, c-b)} \\
& \quad \times \int_{U>0} \frac{\operatorname{det}(U)^{b-(m+1) / 2}}{\operatorname{det}\left(I_{m}+U\right)^{c}} \\
& \quad \times \operatorname{etr}\left[X\left(I_{m}+U\right)^{-1} U-\Sigma\left(U+U^{-1}\right)\right] d U,
\end{aligned}
$$

where $X$ and $\Sigma$ are $m \times m$ symmetric matrices with $\Sigma \geq 0$.

Proof. In the integral of the ECHFMA given in (61), consider the transformation $Z=\left(I_{m}+U\right)^{-1} U$, whose Jacobian is $J(Z \rightarrow U)=\operatorname{det}\left(I_{m}+U\right)^{-(m+1)}$.

If we take $\Sigma=0$ in (65), we arrive at representation (25) of the classical confluent hypergeometric function of matrix argument.

Theorem 13. Let $A, C, \Sigma$, and $X$ be $m \times m$ symmetric matrices with $C-A>0$ and $\Sigma \geq 0$. If $\operatorname{Re}(b)>(m-1) / 2$ and $\operatorname{Re}(c-b)>$ $(m-1) / 2$, then

$$
\begin{aligned}
& \Phi_{\Sigma}(b ; c ; X) \\
& =\frac{\operatorname{det}(C-A)^{-c+(m+1) / 2}}{B_{m}(b, c-b)} \\
& \quad \times \int_{A<U<C} \operatorname{det}(U-A)^{b-(m+1) / 2} \\
& \quad \times \operatorname{det}(C-U)^{c-b-(m+1) / 2} \\
& \quad \times \operatorname{etr}\left[X(C-A)^{-1 / 2}(U-A)(C-A)^{-1 / 2}\right] \\
& \quad \times \operatorname{etr}\left[-\Sigma_{1}(U-A)^{-1}(C-A)(C-U)^{-1}\right] d U,
\end{aligned}
$$

where $\Sigma_{1}=(C-A)^{1 / 2} \Sigma(C-A)^{1 / 2}$.

Proof. Transforming $Z=(C-A)^{-1 / 2}(U-A)(C-A)^{-1 / 2}$ with the Jacobian $J(Z \rightarrow U)=\operatorname{det}(C-A)^{-(m+1) / 2}$ in representation (61), we obtain the result.

If we consider $C=I_{m}$ and $A=-I_{m}$ in the above theorem, then we have

$$
\begin{aligned}
& \Phi_{\Sigma}(b ; c ; X) \\
& =\frac{2^{-m(c-(m+1) / 2)} \operatorname{etr}(X / 2)}{B_{m}(b, c-b)}
\end{aligned}
$$

$$
\begin{aligned}
\times \int_{-I_{m}<U<I_{m}} & \operatorname{det}\left(I_{m}+U\right)^{b-(m+1) / 2} \\
& \times \operatorname{det}\left(I_{m}-U\right)^{c-b-(m+1) / 2} \\
& \times \operatorname{etr}\left[\frac{X U}{2}-4 \Sigma\left(I_{m}+U\right)^{-1}\left(I_{m}-U\right)^{-1}\right] d U .
\end{aligned}
$$

Theorem 14. Let $X$ and $\Sigma$ be $m \times m$ symmetric matrices, $\Sigma>0$. If $b>(m-1) / 2$ and $c-b>(m-1) / 2$, then

$$
\Phi_{\Sigma}(b ; c ; X) \leq \operatorname{etr}(-4 \Sigma) \Phi(b ; c ; X) \leq \frac{\exp (-m)}{\operatorname{det}(4 \Sigma)} \Phi(b ; c ; X) .
$$

Proof. The proof is similar to the proof of Theorem 5.

The $M$-transform of the extended matrix variate confluent hypergeometric function is given next.

Theorem 15. If $X$ is an $m \times m$ symmetric matrix, $\operatorname{Re}(s)>(m-$ $1) / 2, \operatorname{Re}(b)>(m-1) / 2$, and $\operatorname{Re}(c-b)>(m-1) / 2$, then

$$
\begin{aligned}
\int_{\Sigma>0} \operatorname{det}(\Sigma)^{s-(m+1) / 2} \Phi_{\Sigma}(b ; c ; X) d \Sigma \\
\quad=\frac{\Gamma_{m}(s) B_{m}(b+s, c-b+s)}{B_{m}(b, c-b)} \Phi(b+s ; c+2 s ; X) .
\end{aligned}
$$

Proof. Replacing $\Phi_{\Sigma}(b ; c ; X)$ by its equivalent integral representation given in (61) and changing the order of integration, the integral in (69) is rewritten as

$$
\begin{aligned}
& \int_{\Sigma>0} \operatorname{det}(\Sigma)^{s-(m+1) / 2} \Phi_{\Sigma}(b ; c ; X) d \Sigma \\
& =\frac{1}{B_{m}(b, c-b)} \\
& \quad \times \int_{0<Z<I_{m}} \int_{\Sigma>0} \operatorname{det}(\Sigma)^{s-(m+1) / 2} \\
& \quad \times \operatorname{etr}\left[-\Sigma Z^{-1}\left(I_{m}-Z\right)^{-1}\right] d \Sigma \\
& \quad \times \operatorname{det}(Z)^{b-(m+1) / 2} \\
& \quad \times \operatorname{det}\left(I_{m}-Z\right)^{c-b-(m+1) / 2} \operatorname{etr}(X Z) d Z \\
& =\frac{\Gamma_{m}(s)}{B_{m}(b, c-b)} \int_{0<Z<I_{m}} \operatorname{det}(Z)^{b+s-(m+1) / 2} \\
& \quad \times \operatorname{det}\left(I_{m}-Z\right)^{c-b+s-(m+1) / 2} \operatorname{etr}(X Z) d Z,
\end{aligned}
$$

where the last line has been obtained by using (18). Finally, evaluating (70) using the definition of the confluent hypergeometric function of matrix argument, we get the desired result. 
By putting $s=(m+1) / 2$, in (69), we get an interesting relation:

$$
\begin{aligned}
\int_{\Sigma>0} & \Phi_{\Sigma}(b ; c ; X) d \Sigma \\
= & \frac{\Gamma_{m}[(m+1) / 2] B_{m}(b+(m+1) / 2, c-b+(m+1) / 2)}{B_{m}(b, c-b)} \\
& \times \Phi\left(b+\frac{m+1}{2} ; c+m+1 ; X\right) .
\end{aligned}
$$

\section{Extended Whittaker Function of Matrix Argument}

This section gives the definition of the extended Whittaker function of matrix argument, which is a generalization of the Whittaker function of matrix argument given in (32). Several properties and integral representations of this function are also derived.

Definition 16. The extended Whittaker function of matrix argument (EWFMA), denoted by $M_{\Sigma}(k, \mu ; X)$, is defined for an $m \times m$ symmetric matrix $X$ as

$$
\begin{aligned}
M_{\Sigma}(k, \mu ; X)= & \operatorname{det}(X)^{\mu+(m+1) / 4} \operatorname{etr}\left(-\frac{1}{2} X\right) \\
& \times \Phi_{\Sigma}\left(\mu-k+\frac{m+1}{4} ; 2 \mu+\frac{m+1}{2} ; X\right),
\end{aligned}
$$

where $\operatorname{Re}(\mu \pm k)>(m-3) / 4$ and $\Sigma \geq 0$.

If we consider $\Sigma=0$ in (72), then the extended Whittaker function of matrix argument reduces to the classical Whittaker function of matrix argument given in (32); that is, $M_{0}(k, \mu ; X)=M(k, \mu ; X)$. Several properties of the extended Whittaker function of matrix argument are inherited from the ECHFMA, so, as a consequence of Theorem 9, we have

$$
M_{H \Sigma H^{\prime}}\left(k, \mu ; \lambda I_{m}\right)=M_{\Sigma}\left(k, \mu ; \lambda I_{m}\right), \quad \lambda \in \mathbb{R}, H \in O(m)
$$

which indicates that the function $M_{\Sigma}\left(k, \mu ; \lambda I_{m}\right)$ with $\lambda \epsilon$ $\mathbb{R}$ depends on the matrix $\Sigma$ only through its eigenvalues. Similarly,

$$
M_{\sigma I_{m}}\left(k, \mu ; H X H^{\prime}\right)=M_{\sigma I_{m}}(k, \mu ; X), \quad \sigma \geq 0, H \in O(m) .
$$

The above equation means that $M_{\sigma I_{m}}(k, \mu ; X)$ with $\sigma \geq 0$ depends on the matrix $X$ only through its eigenvalues.

An integral representation for the extended Whittaker function of matrix argument $M_{\Sigma}(k, \mu ; X)$ is obtained by replacing in (72) the integral representation of ECHFMA given in (61). In fact,

$$
\begin{aligned}
& M_{\Sigma}(k, \mu ; X) \\
& =\frac{\operatorname{det}(X)^{\mu+(m+1) / 4} \operatorname{etr}(-X / 2)}{B_{m}(\mu-k+(m+1) / 4, \mu+k+(m+1) / 4)} \\
& \quad \times \int_{0<Z<I_{m}} \operatorname{etr}\left[X Z-\Sigma Z^{-1}\left(I_{m}-Z\right)^{-1}\right] \\
& \quad \quad \times \operatorname{det}(Z)^{\mu-k-(m+1) / 4} \operatorname{det}\left(I_{m}-Z\right)^{\mu+k-(m+1) / 4} d Z .
\end{aligned}
$$

Likewise, substitution of (67) in (72) yields the representation

$$
\begin{aligned}
& M_{\Sigma}(k, \mu ; X) \\
& =\frac{2^{-2 m \mu} \operatorname{det}(X)^{\mu+(m+1) / 4}}{B_{m}(\mu-k+(m+1) / 4, \mu+k+(m+1) / 4)} \\
& \quad \times \int_{-I_{m}<U<I_{m}} \operatorname{det}\left(I_{m}+U\right)^{\mu-k-(m+1) / 4} \\
& \quad \times \operatorname{det}\left(I_{m}-U\right)^{\mu+k-(m+1) / 4} \\
& \quad \times \operatorname{etr}\left[\frac{X U}{2}-4 \Sigma\left(I_{m}+U\right)^{-1}\left(I_{m}-U\right)^{-1}\right] d U .
\end{aligned}
$$

Clearly, when we take $\Sigma=0$ in the above expression, we obtain the integral representation (33) of the classical Whittaker function of matrix argument.

Theorem 17. For $m \times m$ symmetric matrix $X$,

$$
M_{\Sigma}(k, \mu ;-X)=(-1)^{m[\mu+(m+1) / 4]} M_{\Sigma}(-k, \mu ; X),
$$

where $\Sigma \geq 0$ and $\operatorname{Re}(\mu \pm k)>(m-3) / 4$.

Proof. Using transformation (64) in (72), we have

$$
\begin{aligned}
M_{\Sigma}(k, \mu ;-X)= & (-1)^{m[\mu+(m+1) / 4]} \\
& \times \operatorname{det}(X)^{\mu+(m+1) / 4} \operatorname{etr}\left(-\frac{1}{2} X\right) \\
& \times \Phi_{\Sigma}\left(\mu+k+\frac{m+1}{4} ; 2 \mu+\frac{m+1}{2} ; X\right) .
\end{aligned}
$$

Substituting (72) in the previous expression gives the result.

Theorem 18. If $\Sigma>0$ and $\operatorname{Re}(\mu \pm k)>(m-3) / 4$, then

$$
\begin{aligned}
M_{\Sigma}(k, \mu ; X) & \leq \operatorname{etr}(-4 \Sigma) M(k, \mu ; X) \\
& \leq \frac{\exp (-m)}{\operatorname{det}(4 \Sigma)} M(k, \mu ; X) .
\end{aligned}
$$

Proof. The result follows by using inequality (68) in (72). 
The following theorem gives the $M$-transform of the extended Whittaker function of matrix argument.

Theorem 19. If $X$ is an $m \times m$ symmetric matrix, $\operatorname{Re}(\mu \pm k)>$ $(m-3) / 4$, and $\operatorname{Re}(s)>(m-1) / 2$, then

$$
\begin{aligned}
& \int_{\Sigma>0} \operatorname{det}(\Sigma)^{s-(m+1) / 2} M_{\Sigma}(k, \mu ; X) d \Sigma \\
& =\frac{\Gamma_{m}(s) B_{m}(\mu-k+s+(m+1) / 4, \mu+k+s+(m+1) / 4)}{B_{m}(\mu-k+(m+1) / 4, \mu+k+(m+1) / 4)} \\
& \quad \times \operatorname{det}(X)^{-s} M(k, \mu+s ; X) .
\end{aligned}
$$

Proof. Writing $M_{\Sigma}(k, \mu, X)$ in terms of $\Phi_{\Sigma}$ using (72), one obtains

$$
\begin{aligned}
& \int_{\Sigma>0} \operatorname{det}(\Sigma)^{s-(m+1) / 2} M_{\Sigma}(k, \mu ; X) d \Sigma \\
& =\operatorname{det}(X)^{\mu+(m+1) / 4} \operatorname{etr}\left(-\frac{1}{2} X\right) \int_{\Sigma>0} \operatorname{det}(\Sigma)^{s-(m+1) / 2} \\
& \quad \times \Phi_{\Sigma}\left(\mu-k+\frac{m+1}{4} ; 2 \mu+\frac{m+1}{2} ; X\right) d \Sigma
\end{aligned}
$$

Now, calculating the above integral by using (69) and then substituting the resulting expression in terms of Whittaker function of matrix argument, we get the final result.

Substitution of $s=(m+1) / 2$ in the above theorem yields an interesting relationship between $M_{\Sigma}(k, \mu ; X)$ and $M(k, \mu ; X)$ as

$$
\begin{aligned}
& \int_{\Sigma>0} M_{\Sigma}(k, \mu ; X) d \Sigma \\
& =\frac{\Gamma_{m}[(m+1) / 2] B_{m}(\mu-k+3(m+1) / 4, \mu+k+3(m+1) / 4)}{B_{m}(\mu-k+(m+1) / 4, \mu+k+(m+1) / 4)} \\
& \quad \times \operatorname{det}(X)^{-(m+1) / 2} M\left(k, \mu+\frac{m+1}{2} ; X\right) .
\end{aligned}
$$

\section{Relationship between EGHFMA, ECHFMA, and EWFMA}

In this section, we derive some results that are related to EGHFMA, ECHFMA, and EWFMA.

Theorem 20. Let $\Sigma, A$, and $B$ be $m \times m$ symmetric matrices such that $\Sigma \geq 0, A>0, B>0$, and $A-B \geq 0$. If $\operatorname{Re}(a)>$ $(m-1) / 2, \operatorname{Re}(b)>(m-1) / 2$, and $\operatorname{Re}(c-b)>(m-1) / 2$, then

$$
\begin{aligned}
& \int_{X>0} \operatorname{etr}(-A X) \operatorname{det}(X)^{a-(m+1) / 2} \Phi_{\Sigma}\left(b ; c ; B^{1 / 2} X B^{1 / 2}\right) d X \\
& =\Gamma_{m}(a) \operatorname{det}(A)^{-a} F_{\Sigma}\left(a, b ; c ; B^{1 / 2} A^{-1} B^{1 / 2}\right) .
\end{aligned}
$$

Proof. Using the integral representation (61) and changing the order of integration, we have

$$
\begin{aligned}
& \int_{X>0} \operatorname{det}(X)^{a-(m+1) / 2} \operatorname{etr}(-A X) \Phi_{\Sigma}\left(b ; c ; B^{1 / 2} X B^{1 / 2}\right) d X \\
& =\frac{1}{B_{m}(b, c-b)} \\
& \quad \times \int_{0<Z<I_{m}} \operatorname{etr}\left[-\Sigma Z^{-1}\left(I_{m}-Z\right)^{-1}\right] \\
& \quad \times \operatorname{det}(Z)^{b-(m+1) / 2} \operatorname{det}\left(I_{m}-Z\right)^{c-b-(m+1) / 2} \\
& \quad \times \int_{X>0} \operatorname{det}(X)^{a-(m+1) / 2} \operatorname{etr}\left[-\left(A-B^{1 / 2} Z B^{1 / 2}\right) X\right] d X d Z .
\end{aligned}
$$

Now, by virtue of (18), we have

$$
\begin{aligned}
& \int_{X>0} \operatorname{det}(X)^{a-(m+1) / 2} \operatorname{etr}(-A X) \Phi_{\Sigma}\left(b ; c ; B^{1 / 2} X B^{1 / 2}\right) d X \\
& =\frac{\Gamma_{m}(a) \operatorname{det}(A)^{-a}}{B_{m}(b, c-b)} \\
& \quad \times \int_{0<Z<I_{m}} \operatorname{etr}\left[-\sum Z^{-1}\left(I_{m}-Z\right)^{-1}\right] \\
& \quad \times \frac{\operatorname{det}(Z)^{b-(m+1) / 2} \operatorname{det}\left(I_{m}-Z\right)^{c-b-(m+1) / 2} d Z}{\operatorname{det}\left(I_{m}-B^{1 / 2} A^{-1} B^{1 / 2} Z\right)^{a}} .
\end{aligned}
$$

Finally, we use (40) to achieve the final result.

Corollary 21. Let $\Sigma$ and $A$ be $m \times m$ symmetric matrices such that $\Sigma \geq 0$ and $A>0$. If $\operatorname{Re}(a)>(m-1) / 2, \operatorname{Re}(b)>(m-1) / 2$, and $\operatorname{Re}(c-b)>(m-1) / 2$, then

$$
\begin{gathered}
\int_{X>0} \operatorname{det}(X)^{a-(m+1) / 2} \Phi_{\Sigma}\left(b ; c ;-A^{1 / 2} X A^{1 / 2}\right) d X \\
=\frac{\Gamma_{m}(a) B_{m}(b-a, c-b ; \Sigma)}{B_{m}(b, c-b)} \operatorname{det}(A)^{-a} .
\end{gathered}
$$

Proof. Application of transformation (64) yields

$$
\begin{gathered}
\int_{X>0} \operatorname{det}(X)^{a-(m+1) / 2} \Phi_{\Sigma}\left(b ; c ;-A^{1 / 2} X A^{1 / 2}\right) d X \\
=\int_{X>0} \operatorname{etr}(-A X) \operatorname{det}(X)^{a-(m+1) / 2} \\
\times \Phi_{\Sigma}\left(c-b ; c ; A^{1 / 2} X A^{1 / 2}\right) d X .
\end{gathered}
$$

Evaluating the above integral by applying (83) and then using (41), we get the result. 
Corollary 22. Let $\Sigma$ and $A$ be $m \times m$ symmetric matrices such that $\Sigma>0$ and $A>0$. If $\operatorname{Re}(b)>(m-1) / 2, \operatorname{Re}(c)>(m-1) / 2$, and $\operatorname{Re}(c-b)>(m-1) / 2$, then

$$
\begin{array}{r}
\int_{X>0} \operatorname{det}(X)^{c-(m+1) / 2} \Phi_{\Sigma}\left(b ; c ;-A^{1 / 2} X A^{1 / 2}\right) d X \\
=\frac{2^{m} \Gamma_{m}(c) \operatorname{etr}(-2 \Sigma) \operatorname{det}(A)^{-c} K_{b-c}(2 \Sigma)}{B_{m}(b, c-b)} .
\end{array}
$$

Proof. Just take $a=c$ in (86) and then use (36).

Theorem 23. Let $\sum$ and $A$ be $m \times m$ symmetric matrices such that $\Sigma \geq 0$ and $A<I_{m}$. If $\operatorname{Re}(b)>(m-1) / 2$ and $\operatorname{Re}(c-b)>$ $(m-1) / 2$, then

$$
\lim _{a \rightarrow \infty} F_{\Sigma}\left(a, b ; c ; \frac{X}{a}\right)=\Phi_{\Sigma}(b ; c ; X)
$$

Proof. Writing $F_{\Sigma}(a, b ; c ; X / a)$ in terms of integral representation by using (40), taking $a \rightarrow \infty$, and applying the result

$$
\lim _{a \rightarrow \infty} \operatorname{det}\left(I_{m}-\frac{X Z}{a}\right)^{-a}=\operatorname{etr}(X Z),
$$

we obtain the desired result.

Theorem 24. Let $\Sigma$ and $X$ be $m \times m$ symmetric matrices such that $\Sigma \geq 0$ and $X<I_{m}$. If $\operatorname{Re}(d)>(m-1) / 2, \operatorname{Re}(a-d)>$ $(m-1) / 2, \operatorname{Re}(b)>(m-1) / 2$, and $\operatorname{Re}(c-b)>(m-1) / 2$, then

$$
\begin{aligned}
& \int_{0<X<I_{m}} \operatorname{det}(X)^{d-(m+1) / 2} \operatorname{det}\left(I_{m}-X\right)^{a-d-(m+1) / 2} \\
& \quad \times F_{\Sigma}\left(a, b ; c ; A^{1 / 2} X A^{1 / 2}\right) d X \\
& =B_{m}(d, a-d) F_{\Sigma}(d, b ; c ; A) .
\end{aligned}
$$

Proof. Using (40) and changing the order of integration, we obtain

$$
\begin{aligned}
& \int_{0<X<I_{m}} \operatorname{det}(X)^{d-(m+1) / 2} \operatorname{det}\left(I_{m}-X\right)^{a-d-(m+1) / 2} \\
& \quad \times F_{\Sigma}\left(a, b ; c ; A^{1 / 2} X A^{1 / 2}\right) d X \\
& =\frac{1}{B_{m}(b, c-b)} \\
& \times \int_{0<Z<I_{m}} \operatorname{etr}\left[-\sum Z^{-1}\left(I_{m}-Z\right)^{-1}\right] \\
& \quad \times \int_{0<X<I_{m}} \frac{\operatorname{det}(Z)^{b-(m+1) / 2} \operatorname{det}\left(I_{m}-Z\right)^{c-b-(m+1) / 2}}{\operatorname{det}\left(I_{m}-A^{1 / 2} Z A^{1 / 2} X\right)^{a}} d X d Z .
\end{aligned}
$$

Now, evaluating the integral involving $X$ using (22) and applying (28), we have

$$
\begin{aligned}
& \int_{0<X<I_{m}} \operatorname{det}(X)^{d-(m+1) / 2} \operatorname{det}\left(I_{m}-X\right)^{a-d-(m+1) / 2} \\
& \quad \times F_{\Sigma}\left(a, b ; c ; A^{1 / 2} X A^{1 / 2}\right) d X \\
& =\frac{B_{m}(d, a-d)}{B_{m}(b, c-b)} \\
& \quad \times \int_{0<Z<I_{m}}^{\operatorname{etr}\left[-\sum Z^{-1}\left(I_{m}-Z\right)^{-1}\right]} \\
& \quad \times \frac{\operatorname{det}(Z)^{b-(m+1) / 2} \operatorname{det}\left(I_{m}-Z\right)^{c-b-(m+1) / 2} d Z}{\operatorname{det}\left(I_{m}-A Z\right)^{d}} .
\end{aligned}
$$

Finally, using representation (40), we arrive at the desired result.

Corollary 25. For $\Sigma>0, \operatorname{Re}(d)>(m-1) / 2, \operatorname{Re}(a-d)>$ $(m-1) / 2, \operatorname{Re}(b)>(m-1) / 2$, and $\operatorname{Re}(c-b)>(m-1) / 2$, we have

$$
\begin{aligned}
\int_{0<X<I_{m}} \operatorname{det}(X)^{d-(m+1) / 2} \operatorname{det}\left(I_{m}-X\right)^{a-d-(m+1) / 2} \\
\quad \times F_{\Sigma}(a, b ; c ; X) d X \\
=B_{m}(d, a-d) F_{\Sigma}\left(d, b ; c ; I_{m}\right) \\
=\frac{B_{m}(d, a-d) B_{m}(b, c-b-d ; \Sigma)}{B_{m}(b, c-b)}
\end{aligned}
$$

Proof. Just take $A=I_{m}$ in (91) and then use (41).

Corollary 26. For $\Sigma>0, \operatorname{Re}(c)>(m-1) / 2, \operatorname{Re}(a-c)>$ $(m-1) / 2, \operatorname{Re}(b)>(m-1) / 2$, and $\operatorname{Re}(c-b)>(m-1) / 2$, we have

$$
\begin{aligned}
\int_{0<X<I_{m}} \operatorname{det}(X)^{c-(m+1) / 2} \operatorname{det}\left(I_{m}-X\right)^{a-c-(m+1) / 2} \\
\quad \times F_{\Sigma}(a, b ; c ; X) d X \\
=\frac{2^{m} B_{m}(c, a-c) \operatorname{etr}(-2 \Sigma) K_{b}(2 \Sigma)}{B_{m}(b, c-b)}
\end{aligned}
$$

Proof. Just take $c=d$ in (94) and then use (36).

Corollary 27. For $\Sigma>0, \operatorname{Re}(d)>(m-1) / 2, \operatorname{Re}(a-d)>$ $(m-1) / 2, \operatorname{Re}(b)>(m-1) / 2$, and $\operatorname{Re}(c-b)>(m-1) / 2$, we have

$$
\begin{aligned}
\int_{0<X<I_{m}} \operatorname{det}(X)^{d-(m+1) / 2} \operatorname{det}\left(I_{m}-X\right)^{a-d-(m+1) / 2} \\
\quad \times F_{\Sigma}\left(a, b ; c ; I_{m}-X\right) d X \\
=\frac{B_{m}(d, a-d) B_{m}(b, c+d-a-b ; \Sigma)}{B_{m}(b, c-b)} .
\end{aligned}
$$

Proof. The proof follows from Corollary 25. 
Theorem 28. Let $\Sigma$ and $A$ be $m \times m$ symmetric matrices such that $\Sigma>0$ and $A>0$. If $\operatorname{Re}(b)>(m-1) / 2, \operatorname{Re}(d)>(m-1) / 2$, $\operatorname{Re}(a-d)>(m-1) / 2$, and $\operatorname{Re}(c-b)>(m-1) / 2$, then

$$
\begin{gathered}
\int_{X>0} \operatorname{det}(X)^{d-(m+1) / 2} F_{\Sigma}\left(a, b ; c ;-A^{1 / 2} X A^{1 / 2}\right) d X \\
=\frac{\operatorname{det}(A)^{-d} B_{m}(d, a-d) B_{m}(b-d, c-b ; \Sigma)}{B_{m}(b, c-b)} .
\end{gathered}
$$

Proof. Using representation (40) and changing the order of integration, we get

$$
\begin{aligned}
& \int_{X>0} \operatorname{det}(X)^{d-(m+1) / 2} F_{\Sigma}\left(a, b ; c ;-A^{1 / 2} X A^{1 / 2}\right) d X \\
& =\frac{1}{B_{m}(b, c-b)} \\
& \quad \times \int_{0<Z<I_{m}} \operatorname{det}(Z)^{b-(m+1) / 2} \\
& \quad \times \operatorname{det}\left(I_{m}-Z\right)^{c-b-(m+1) / 2} \operatorname{etr}\left[-\Sigma Z^{-1}\left(I_{m}-Z\right)^{-1}\right] \\
& \quad \times \int_{X>0} \frac{\operatorname{det}(X)^{d-(m+1) / 2}}{\operatorname{det}\left(I_{m}+A^{1 / 2} Z A^{1 / 2} X\right)^{a}} d X d Z .
\end{aligned}
$$

Now, using (20) to integrate with respect to $X$, we get

$$
\begin{aligned}
& \int_{X>0} \operatorname{det}(X)^{d-(m+1) / 2} F_{\Sigma}\left(a, b ; c ;-A^{1 / 2} X A^{1 / 2}\right) d X \\
&= \frac{\operatorname{det}(A)^{-d} B_{m}(d, a-d)}{B_{m}(b, c-b)} \\
& \quad \times \int_{0<Z<I_{m}} \operatorname{det}(Z)^{b-d-(m+1) / 2} \operatorname{det}\left(I_{m}-Z\right)^{c-b-(m+1) / 2} \\
& \quad \times \operatorname{etr}\left[-\sum Z^{-1}\left(I_{m}-Z\right)^{-1}\right] d Z .
\end{aligned}
$$

Finally, we use (34) to obtain the result.

Corollary 29. Let $\Sigma$ and $A$ be $m \times m$ symmetric matrices such that $\Sigma>0$ and $A>0$. If $\operatorname{Re}(b)>(m-1) / 2, \operatorname{Re}(c)>(m-1) / 2$, $\operatorname{Re}(a-c)>(m-1) / 2$, and $\operatorname{Re}(c-b)>(m-1) / 2$, then

$$
\begin{gathered}
\int_{X>0} \operatorname{det}(X)^{c-(m+1) / 2} F_{\Sigma}\left(a, b ; c ;-A^{1 / 2} X A^{1 / 2}\right) d X \\
=\frac{2^{m} B_{m}(c, a-c) \operatorname{det}(A)^{-c} \operatorname{etr}(-2 \Sigma) K_{b-c}(2 \Sigma)}{B_{m}(b, c-b)} .
\end{gathered}
$$

Proof. Just take $c=d$ in (97) and use relation (36).
Theorem 30. Let $\Sigma, A$, and $B$ be $m \times m$ symmetric matrices such that $\Sigma \geq 0, A>0, B>0$, and $A-B / 2>0$. If $\operatorname{Re}(\mu \pm k)>$ $(m-3) / 4$ and $\operatorname{Re}(\mu+a)>(m-3) / 4$, then

$$
\begin{gathered}
\int_{X>0} \operatorname{etr}(-A X) \operatorname{det}(X)^{a-(m+1) / 2} M_{\Sigma}\left(k, \mu ; B^{1 / 2} X B^{1 / 2}\right) d X \\
=\frac{\Gamma_{m}(a+\mu+(m+1) / 4) \operatorname{det}(B)^{\mu+(m+1) / 4}}{\operatorname{det}(A+B / 2)^{a+\mu+(m+1) / 4}} \\
\quad \times F_{\Sigma}\left(a+\mu+\frac{m+1}{4}, \mu-k+\frac{m+1}{4} ;\right. \\
\left.2 \mu+\frac{m+1}{2} ; 2\left(I_{m}+2 B^{-1 / 2} A B^{-1 / 2}\right)^{-1}\right) .
\end{gathered}
$$

Proof. Using the definition of the extended Whittaker function of matrix argument given in (72), we have

$$
\begin{aligned}
& \int_{X>0} \operatorname{etr}(-A X) \operatorname{det}(X)^{a-(m+1) / 2} M_{\Sigma}\left(k, \mu ; B^{1 / 2} X B^{1 / 2}\right) d X \\
& =\operatorname{det}(B)^{\mu+(m+1) / 4} \\
& \quad \times \int_{X>0} \operatorname{det}(X)^{a+\mu-(m+1) / 4} \operatorname{etr}\left[-\left(A+\frac{1}{2} B\right) X\right] \\
& \quad \times \Phi_{\Sigma}\left(\mu-k+\frac{m+1}{4} ; 2 \mu+\frac{m+1}{2} ; B^{1 / 2} X B^{1 / 2}\right) d X .
\end{aligned}
$$

Now, we use (83) in the above integral to obtain the desired result.

Corollary 31. Let $\Sigma$ and $A$ be $m \times m$ symmetric matrices such that $\Sigma>0$ and $A>0$. If $\operatorname{Re}(\mu \pm k)>(m-3) / 4$ and $\operatorname{Re}(\mu+a)>$ $(m-3) / 4$, then

$$
\begin{aligned}
& \int_{X>0} \operatorname{etr}(-A X) \operatorname{det}(X)^{a-(m+1) / 2} M_{\Sigma}\left(k, \mu ; 2 A^{1 / 2} X A^{1 / 2}\right) d X \\
& =\frac{\Gamma_{m}[a+\mu+(m+1) / 4] B_{m}(\mu-k+(m+1) / 4, k-a ; \Sigma)}{B_{m}(\mu-k+(m+1) / 4, \mu+k+(m+1) / 4) \operatorname{det}(2 A)^{a}} .
\end{aligned}
$$

Proof. Take $B=2 A$ in (101) and then use (41) in the resulting expression.

Corollary 32. Let $\Sigma$ and $A$ be $m \times m$ symmetric matrices such that $\Sigma>0$ and $A>0$. If $\operatorname{Re}(\mu)>-1 / 2$ and $\operatorname{Re}(\mu \pm k)>$ $(m-3) / 4$, then

$$
\begin{aligned}
\int_{X>0} \operatorname{etr}(-A X) \operatorname{det}(X)^{\mu-(m+1) / 4} M_{\Sigma}\left(k, \mu ; 2 A^{1 / 2} X A^{1 / 2}\right) d X \\
=\frac{2^{m} \operatorname{etr}(-2 \Sigma) \Gamma_{m}[2 \mu+(m+1) / 2] \operatorname{det}(2 A)^{-(\mu+(m+1) / 4)}}{B_{m}(\mu-k+(m+1) / 4, \mu+k+(m+1) / 4)} \\
\quad \times K_{\mu-k+(m+1) / 4}(2 \Sigma) .
\end{aligned}
$$

Proof. Take $a=\mu+(m+1) / 4$ in (103) and then use (36) to get the result. 


\section{Extended Matrix Variate Gauss Hypergeometric Function Distribution}

This section defines the extended matrix variate Gauss hypergeometric function distribution which is a generalization of the matrix variate Gauss hypergeometric function distribution. We show that this distribution occurs naturally as the distribution of the matrix quotient $V^{-1 / 2} U V^{-1 / 2}$, where the $m \times m$ random matrices $U$ and $V$ are independent, the random matrix $U$ has a matrix beta type 2 distribution, and the random matrix $V$ follows an extended matrix variate beta type 1 distribution.

Definition 33. An $m \times m$ positive definite random matrix $X$ is said to have an extended matrix variate Gauss hypergeometric function distribution with parameters $(\nu, \alpha, \beta, \gamma, \Sigma)$, denoted by $X \sim \mathrm{EGH}_{m}(\nu, \alpha, \beta, \gamma ; \Sigma)$, if its pdf is given by

$$
\begin{aligned}
& \frac{B_{m}(\beta, \gamma-\beta)}{B_{m}(\nu, \alpha-\nu) B_{m}(\beta-\nu, \gamma-\beta ; \Sigma)} \\
& \quad \times \operatorname{det}(X)^{\nu-(m+1) / 2} F_{\Sigma}(\alpha, \beta ; \gamma ;-X), \quad X>0,
\end{aligned}
$$

where $\nu>(m-1) / 2, \beta>(m-1) / 2, \alpha-\nu>(m-1) / 2$, $\gamma-\beta>(m-1) / 2$, and $\Sigma>0$.

Note that the matrix variate Gauss hypergeometric function distribution (see Gupta and Nagar [13]) can be obtained from (105) by substituting $\Sigma=0$ and putting an additional condition $\beta-v>(m-1) / 2$.

Theorem 36 derives the extended matrix variate Gauss hypergeometric function distribution as the distribution of the matrix ratio of two independent random matrices distributed as beta type 2 and extended beta type 1. First, we define the extended matrix variate beta type 1 , matrix variate beta type 1, and the matrix variate beta type 2 distributions. These definitions can be found in Gupta and Nagar [13], Nagar et al. [18], and Nagar and Roldán-Correa [25].

Definition 34. An $m \times m$ random matrix $X$ is said to have an extended matrix variate beta type 1 distribution with parameters $p, q,-\infty<p<\infty,-\infty<q<\infty$, and $\Sigma>0$, denoted by $X \sim \operatorname{EB} 1(m, p, q ; \Sigma)$, if its pdf is given by

$$
\begin{aligned}
& \frac{\operatorname{det}(X)^{p-(m+1) / 2} \operatorname{det}\left(I_{m}-X\right)^{q-(m+1) / 2}}{B_{m}(p, q ; \Sigma)} \\
& \times \operatorname{etr}\left[-\sum X^{-1}\left(I_{m}-X\right)^{-1}\right], \quad 0<X<I_{m} .
\end{aligned}
$$

Note that, for $\Sigma=0$, we take $p>(m-1) / 2$ and $q>(m-$ $1) / 2$ and the extended matrix variate beta type 1 distribution defined by the above density slides to a matrix variate beta type 1 distribution with the pdf

$$
\frac{\operatorname{det}(X)^{p-(m+1) / 2} \operatorname{det}\left(I_{m}-X\right)^{q-(m+1) / 2}}{B_{m}(p, q)}, \quad 0<X<I_{m} .
$$

We will designate this distribution by $X \sim \mathrm{B} 1(m, p, q)$.
Definition 35. An $m \times m$ random matrix $X$ is said to have a matrix beta type 2 distribution with parameters $p(>(m-$ $1) / 2)$ and $q(>(m-1) / 2)$, denoted by $X \sim \mathrm{B} 2(m, p, q)$, if its pdf is given by

$$
\frac{\operatorname{det}(X)^{p-(m+1) / 2} \operatorname{det}\left(I_{m}+X\right)^{-(p+q)}}{B_{m}(p, q)}, \quad X>0 .
$$

Theorem 36. Let $U$ and $V$ be independent random matrices, where $V \sim \mathrm{B} 2(\mathrm{~m}, \nu, \gamma)$ and $U \sim \operatorname{EB} 1(\mathrm{~m}, \alpha, \beta ; \Sigma)$. Then, $X=$ $U^{-1 / 2} V U^{-1 / 2} \sim \mathrm{EGH}_{\mathrm{m}}(\nu, \nu+\gamma, \alpha+\nu, \alpha+\nu+\beta ; \Sigma)$.

Proof. As $U$ and $V$ are independent, by (106) and (108), the joint density of $U$ and $V$ is given by

$$
\begin{aligned}
& \frac{\operatorname{det}(V)^{\nu-(m+1) / 2} \operatorname{det}(U)^{\alpha-(m+1) / 2} \operatorname{det}\left(I_{m}-U\right)^{\beta-(m+1) / 2}}{B_{m}(\nu, \gamma) B_{m}(\alpha, \beta ; \Sigma) \operatorname{det}\left(I_{m}+V\right)^{\nu+\gamma}} \\
& \times \operatorname{etr}\left[-\Sigma U^{-1}\left(I_{m}-U\right)^{-1}\right],
\end{aligned}
$$

where $V>0$ and $0<U<I_{m}$. Using the transformation $X=$ $U^{-1 / 2} V U^{-1 / 2}$, with the Jacobian $J(V \rightarrow X)=\operatorname{det}(U)^{(m+1) / 2}$, we obtain the joint density as

$$
\begin{gathered}
\frac{\operatorname{det}(X)^{\nu-(m+1) / 2}}{B_{m}(\nu, \gamma) B_{m}(\alpha, \beta ; \Sigma)} \operatorname{etr}\left[-\Sigma U^{-1}\left(I_{m}-U\right)^{-1}\right] \\
\times \frac{\operatorname{det}(U)^{\alpha+\nu-(m+1) / 2} \operatorname{det}\left(I_{m}-U\right)^{\beta-(m+1) / 2}}{\operatorname{det}\left(I_{m}+X U\right)^{\nu+\gamma}},
\end{gathered}
$$

where $0<U<I_{m}$ and $X>0$. To find the density of $X$, we integrate the above expression with respect to $U$ to get

$$
\begin{aligned}
& \frac{\operatorname{det}(X)^{\nu-(m+1) / 2}}{B_{m}(\nu, \gamma) B_{m}(\alpha, \beta ; \Sigma)} \\
& \quad \times \int_{0<U<I_{m}} \operatorname{etr}\left[-\Sigma U^{-1}\left(I_{m}-U\right)^{-1}\right] \\
& \quad \times \frac{\operatorname{det}(U)^{\alpha+\nu-(m+1) / 2} \operatorname{det}\left(I_{m}-U\right)^{\beta-(m+1) / 2} d U}{\operatorname{det}\left(I_{m}+X U\right)^{\nu+\gamma}} .
\end{aligned}
$$

Evaluation of the above expression using (40) yields the desired result.

\section{Extended Matrix Variate Confluent Hypergeometric Function Distribution}

This section defines the extended matrix variate confluent hypergeometric function distribution which is a generalization of the matrix variate confluent hypergeometric function type 1 distribution. We study several properties of this new distribution and its relationship with other known matrix variate distributions. We also show that this distribution occurs naturally as the distribution of the matrix quotient 
$U^{-1 / 2} V U^{-1 / 2}$, where the $m \times m$ random matrices $V$ and $U$ are independent, the random matrix $V$ has a matrix variate gamma distribution, and the random matrix $U$ follows an extended matrix variate beta type 1 distribution.

Definition 37. An $m \times m$ positive definite random matrix $X$ is said to have an extended matrix variate confluent hypergeometric function distribution with parameters $(\nu, \alpha, \beta, \Sigma)$, denoted by $X \sim \mathrm{ECH}_{m}(\nu, \alpha, \beta ; \Sigma)$, if its pdf is given by

$$
\frac{B_{m}(\alpha, \beta-\alpha) \operatorname{det}(X)^{\nu-(m+1) / 2} \Phi_{\Sigma}(\alpha ; \beta ;-X)}{\Gamma_{m}(\nu) B_{m}(\alpha-\nu, \beta-\alpha ; \Sigma)}, \quad X>0,
$$

where $v>(m-1) / 2, \alpha>(m-1) / 2, \beta-\alpha>(m-1) / 2$, and $\Sigma>0$.

For $\Sigma=0$ (with an additional condition $\alpha-v>(m-1) / 2)$, (112) reduces to the matrix variate confluent hypergeometric function density (see Gupta and Nagar [13]).

The extended matrix variate confluent hypergeometric function distribution can be derived as the distribution of the matrix quotient of independent gamma and extended beta matrices as given in the following theorem. First we define the matrix variate gamma distribution. The definition of matrix variate gamma distribution can be found in Gupta and Nagar [13] and Iranmanesh et al. [26].

Definition 38. An $m \times m$ random matrix $X$ is said to have a matrix variate gamma distribution with parameters $p(>(m-$ $1) / 2$ ) and $\Sigma>0$, denoted by $X \sim \mathrm{Ga}(m, p, \Sigma)$, if its pdf is given by

$$
\frac{\operatorname{etr}\left(-\Sigma^{-1} X\right) \operatorname{det}(X)^{p-(m+1) / 2}}{\Gamma_{m}(p) \operatorname{det}(\Sigma)^{p}}, \quad X>0 .
$$

Theorem 39. If $U \sim \mathrm{EB} 1(\mathrm{~m}, \mathrm{~b}, \mathrm{c} ; \Sigma)$ and $V \sim \mathrm{Ga}\left(\mathrm{m}, \mathrm{a}, \mathrm{I}_{\mathrm{m}}\right)$ are independent, then $U^{-1 / 2} V U^{-1 / 2} \sim \mathrm{ECH}_{\mathrm{m}}(\mathrm{a}, \mathrm{a}+\mathrm{b}, \mathrm{a}+\mathrm{b}+$ c; $\Sigma$ ).

Proof. As $U$ and $V$ are independent, from (106) and (113), the joint density of $U$ and $V$ is given by

$$
\begin{aligned}
& \frac{\operatorname{det}(V)^{a-(m+1) / 2} \operatorname{det}(U)^{b-(m+1) / 2} \operatorname{det}\left(I_{m}-U\right)^{c-(m+1) / 2}}{\Gamma_{m}(a) B_{m}(b, c ; \Sigma)} \\
& \times \operatorname{etr}\left[-V-\Sigma U^{-1}\left(I_{m}-U\right)^{-1}\right]
\end{aligned}
$$

where $V>0$ and $0<U<I_{m}$. Making the transformation $X=$ $U^{-1 / 2} V U^{-1 / 2}$, with the Jacobian $J(V \rightarrow X)=\operatorname{det}(U)^{(m+1) / 2}$, we find the joint density of $U$ and $X$ as

$$
\begin{aligned}
& \frac{\operatorname{det}(X)^{a-(m+1) / 2} \operatorname{det}(U)^{a+b-(m+1) / 2} \operatorname{det}\left(I_{m}-U\right)^{c-(m+1) / 2}}{\Gamma_{m}(a) B_{m}(b, c ; \Sigma)} \\
& \times \operatorname{etr}\left[-X U-\Sigma U^{-1}\left(I_{m}-U\right)^{-1}\right]
\end{aligned}
$$

where $0<U<I_{m}$ and $X>0$. Now, the density of $X$ is obtained by integrating the above expression with respect to $U$ by using the integral representation (61).
Theorem 40. Let the random variables $U$ and $V$ be independent, $U \sim \mathrm{EB} 1(\mathrm{~m}, \alpha, \beta ; \Sigma)$, and $V \sim \mathrm{B} 2(\mathrm{~m}, \nu, \gamma)$. Then, $Y=U^{1 / 2} V U^{1 / 2}$ has the density

$$
\begin{aligned}
& \frac{B_{m}(\alpha+\gamma, \beta)}{B_{m}(\nu, \gamma) B_{m}(\alpha, \beta ; \Sigma)} \frac{\operatorname{det}(Y)^{\nu-(m+1) / 2}}{\operatorname{det}\left(I_{m}+Y\right)^{\nu+\gamma}} \\
& \quad \times F_{\Sigma}\left(\nu+\gamma, \beta ; \alpha+\beta+\gamma ;\left(I_{m}+Y\right)^{-1}\right), \quad Y>0 .
\end{aligned}
$$

Proof. The joint density of $U$ and $V$ is given in (109). Using the transformation $Y=U^{1 / 2} V U^{1 / 2}$, with the Jacobian $J(V \rightarrow$ $Y)=\operatorname{det}(U)^{-(m+1) / 2}$, we obtain the joint density of $U$ and $Y$ as

$$
\begin{aligned}
& \frac{\operatorname{det}(Y)^{\nu-(m+1) / 2}}{B_{m}(\nu, \gamma) B_{m}(\alpha, \beta ; \Sigma) \operatorname{det}\left(I_{m}+Y\right)^{\nu+\gamma}} \\
& \times \frac{\operatorname{det}(U)^{\alpha+\gamma-(m+1) / 2} \operatorname{det}\left(I_{m}-U\right)^{\beta-(m+1) / 2}}{\operatorname{det}\left(I_{m}-\left(I_{m}+Y\right)^{-1}\left(I_{m}-U\right)\right)^{\nu+\gamma}} \\
& \times \operatorname{etr}\left[-\Sigma U^{-1}\left(I_{m}-U\right)^{-1}\right],
\end{aligned}
$$

where $0<U<I_{m}$ and $Y>0$. Now, integration of $U$ in the above expression by using (40) yields the desired result.

Corollary 41. Let the random variables $U$ and $V$ be independent, $U \sim \mathrm{B} 1(\mathrm{~m}, \alpha, \beta)$, and $V \sim \mathrm{B} 2(\mathrm{~m}, \nu, \gamma)$. Then, $Y=$ $U^{1 / 2} V U^{1 / 2}$ has the density

$$
\begin{aligned}
& \frac{B_{m}(\alpha+\gamma, \beta)}{B_{m}(\nu, \gamma) B_{m}(\alpha, \beta)} \frac{\operatorname{det}(Y)^{\nu-(m+1) / 2}}{\operatorname{det}\left(I_{m}+Y\right)^{\nu+\gamma}} \\
& \quad \times F\left(\nu+\gamma, \beta ; \alpha+\beta+\gamma ;\left(I_{m}+Y\right)^{-1}\right), \quad Y>0 .
\end{aligned}
$$

\section{Conflict of Interests}

The authors declare that there is no conflict of interests regarding the publication of this paper.

\section{Acknowledgment}

This research work was supported by the Sistema Universitario de Investigación, Universidad de Antioquia, by Project no. IN10164CE.

\section{References}

[1] Y. L. Luke, The Special Functions and Their Approximations, vol. 1, Academic Press, New York, NY, USA, 1969.

[2] E. T. Whittaker and G. N. Watson, A Course of Modern Analysis, Cambridge University Press, New York, NY, USA, 1996.

[3] M. A. Chaudhry, A. Qadir, M. Rafique, and S. M. Zubair, "Extension of Euler's beta function," Journal of Computational and Applied Mathematics, vol. 78, no. 1, pp. 19-32, 1997.

[4] A. R. Miller, "Remarks on a generalized beta function," Journal of Computational and Applied Mathematics, vol. 100, no. 1, pp. 23-32, 1998. 
[5] M. A. Chaudhry, A. Qadir, H. M. Srivastava, and R. B. Paris, "Extended hypergeometric and confluent hypergeometric functions," Applied Mathematics and Computation, vol. 159, no. 2, pp. 589-602, 2004.

[6] R. W. Butler and A. T. Wood, "Laplace approximations for hypergeometric functions with matrix argument," Annals of Statistics, vol. 30, no. 4, pp. 1155-1177, 2002.

[7] R. W. Butler and A. T. Wood, "Laplace approximation for Bessel functions of matrix argument," Journal of Computational and Applied Mathematics, vol. 155, no. 2, pp. 359-382, 2003.

[8] R. W. Butler and A. T. Wood, "Laplace approximations to hypergeometric functions of two matrix arguments," Journal of Multivariate Analysis, vol. 94, no. 1, pp. 1-18, 2005.

[9] C. S. Herz, "Bessel functions of matrix argument," Annals of Mathematics, vol. 61, no. 2, pp. 474-523, 1955.

[10] A. G. Constantine, "Some non-central distribution problems in multivariate analysis," Annals of Mathematical Statistics, vol. 34, pp. 1270-1285, 1963.

[11] A. T. James, "Distributions of matrix variates and latent roots derived from normal samples," Annals of Mathematical Statistics, vol. 35, pp. 475-501, 1964.

[12] R. J. Muirhead, Aspects of Multivariate Statistical Theory, Wiley Series in Probability and Mathematical Statistics, John Wiley \& Sons, New York, NY, USA, 1982.

[13] A. K. Gupta and D. K. Nagar, Matrix Variate Distributions, Chapman \& Hall/CRC, Boca Raton, Fla, USA, 2000.

[14] A. Bekker, J. J. Roux, R. Ehlers, and M. Arashi, "Bimatrix variate beta type IV distribution: relation to Wilks's statistic and bimatrix variate Kummer-beta type IV distribution," Communications in Statistics-Theory and Methods, vol. 40, no. 23, pp. 4165-4178, 2011.

[15] A. Bekker, J. J. J. Roux, R. Ehlers, and M. Arashi, "Distribution of the product of determinants of noncentral bimatrix beta variates," Journal of Multivariate Analysis, vol. 109, pp. 73-87, 2012.

[16] A. Bekker, J. J. J. Roux, and M. Arashi, "Wishart ratios with dependent structure: new members of the bimatrix beta type IV," Linear Algebra and Its Applications, vol. 435, no. 12, pp. 3243-3260, 2011.

[17] A. K. Gupta and D. K. Nagar, "Matrix-variate Gauss hypergeometric distribution," Journal of the Australian Mathematical Society, vol. 92, no. 3, pp. 335-355, 2012.

[18] D. K. Nagar, A. Roldán-Correa, and A. K. Gupta, "Extended matrix variate gamma and beta functions," Journal of Multivariate Analysis, vol. 122, pp. 53-69, 2013.

[19] A. E. Ingham, "An integral which occurs in statistics," Mathematical Proceedings of the Cambridge Philosophical Society, vol. 29, pp. 271-276, 1933.

[20] C. L. Siegel, "Uber die analytische theorie der quadratischen formen," Annals of Mathematics, Second Series, vol. 36, no. 3, pp. 527-606, 1935.

[21] H. Hashiguchi, Y. Numata, N. Takayama, and A. Takemura, "The holonomic gradient method for the distribution function of the largest root of a Wishart matrix," Journal of Multivariate Analysis, vol. 117, pp. 296-312, 2013.

[22] P. Koev and A. Edelman, "The efficient evaluation of the hypergeometric function of a matrix argument," Mathematics of Computation, vol. 75, no. 254, pp. 833-846, 2006.

[23] W. H. Abdi, "Whittaker's $M_{k, \mu}$-function of a matrix argument," Rendiconti del Circolo Matematico di Palermo, Serie II, vol. 17, no. 3, pp. 333-342, 1968.
[24] K. M. Abadir and J. R. Magnus, Matrix Algebra, Cambridge University Press, New York, NY, USA, 2005.

[25] D. K. Nagar and A. Roldán-Correa, "Extended matrix variate beta distributions," Progress in Applied Mathematics, vol. 6, no. 1, pp. 40-53, 2013.

[26] A. Iranmanesh, M. Arashi, D. K. Nagar, and S. M. M. Tabatabaey, "On inverted matrix variate gamma distribution," Communications in Statistics. Theory and Methods, vol. 42, no. 1, pp. 28-41, 2013. 


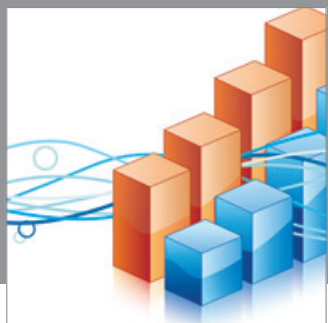

Advances in

Operations Research

mansans

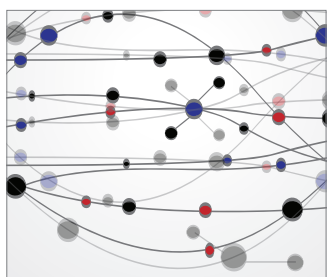

The Scientific World Journal
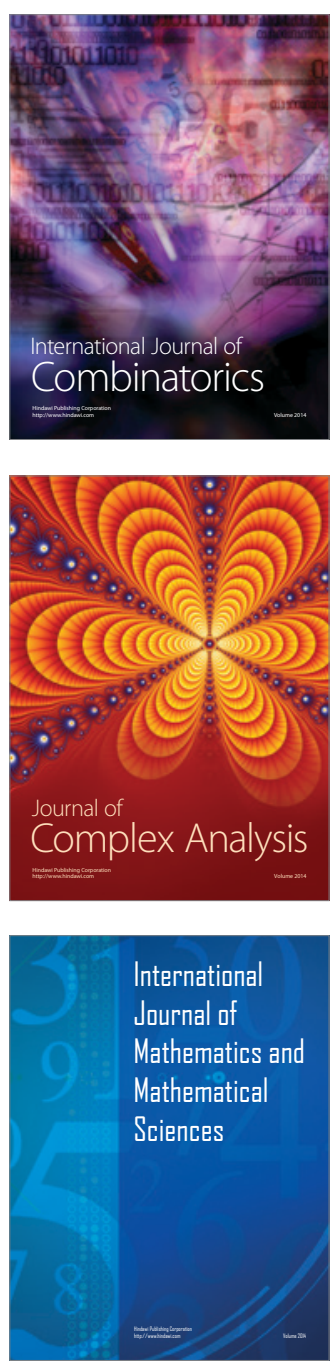
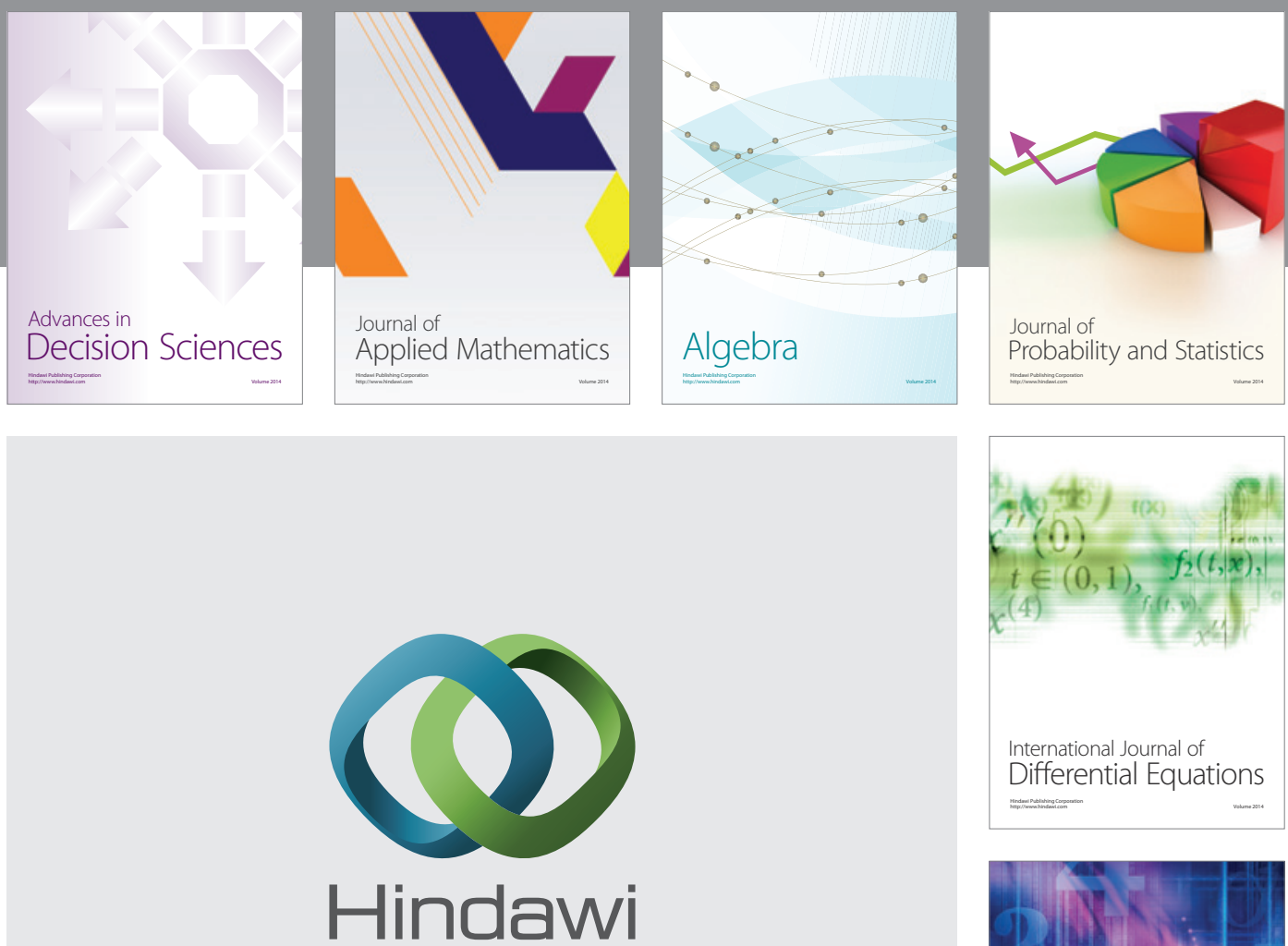

Submit your manuscripts at http://www.hindawi.com
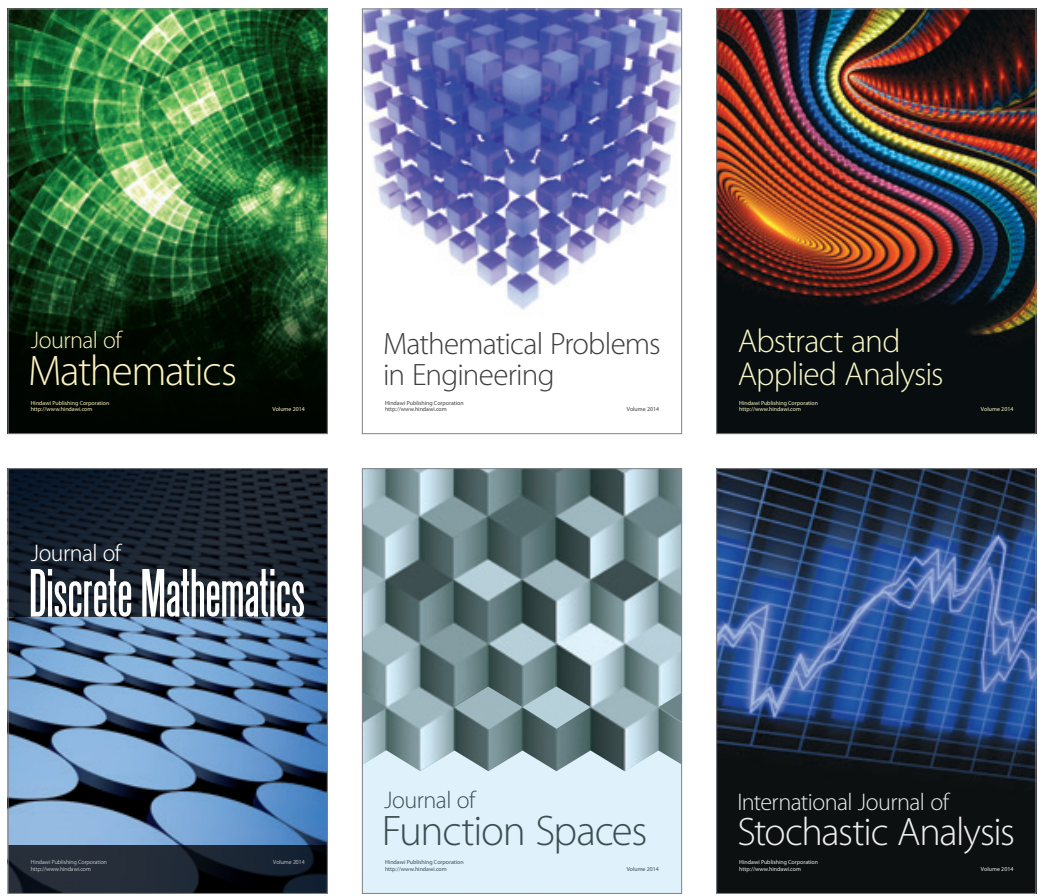

Journal of

Function Spaces

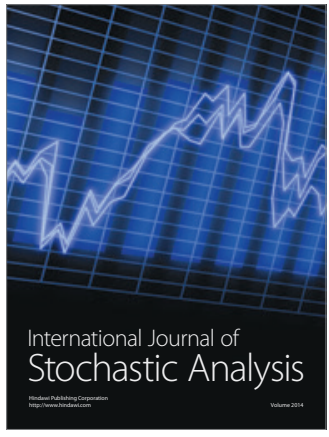

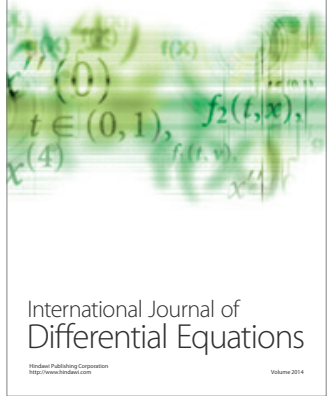
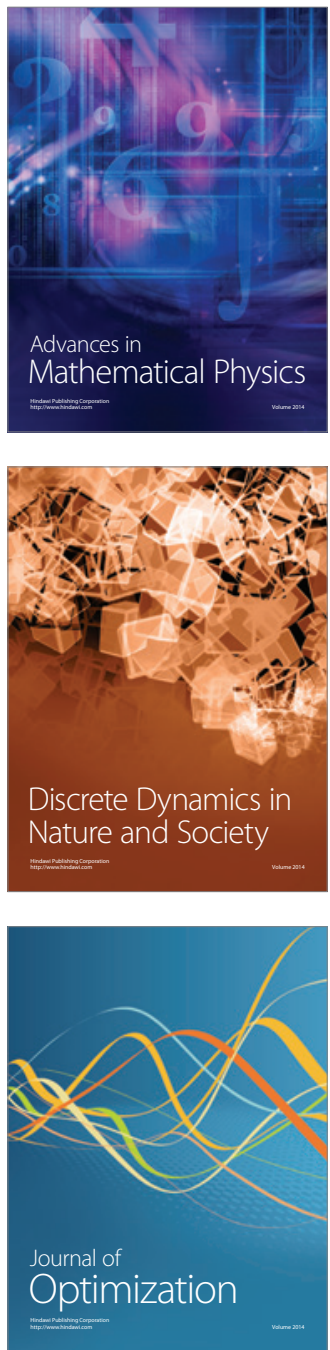